MATHEMATICS OF COMPUTATION

Volume 77, Number 262, April 2008, Pages 851-882

S 0025-5718(07)02054-6

Article electronically published on December 13, 2007

\title{
CONVERGENCE OF A FINITE VOLUME SCHEME FOR COAGULATION-FRAGMENTATION EQUATIONS
}

\author{
JEAN-PIERRE BOURGADE AND FRANCIS FILBET
}

\begin{abstract}
This paper is devoted to the analysis of a numerical scheme for the coagulation and fragmentation equation. A time explicit finite volume scheme is developed, based on a conservative formulation of the equation. It is shown to converge under a stability condition on the time step, while a first order rate of convergence is established and an explicit error estimate is given. Finally, several numerical simulations are performed to investigate the gelation phenomenon and the long time behavior of the solution.
\end{abstract}

\section{INTRODUCTION}

Coagulation and fragmentation processes arise in the dynamics of cluster growth and describe the mechanisms by which clusters can coalesce to form larger clusters or break apart into smaller pieces. In the simplest coagulation-fragmentation models the clusters are usually assumed to be fully identified by their size (or volume, or number of particles). The coagulation-fragmentation models we consider in this paper describe the time evolution of the cluster size distribution as the system of clusters undergoes binary coagulation and binary fragmentation events. More precisely, denoting by $C_{x}$ the clusters of size $x$ with $x \in \mathbb{R}^{+}=(0, \infty)$, the basic reactions taken into account herein are

$$
C_{x}+C_{x^{\prime}} \stackrel{a\left(x, x^{\prime}\right)}{\longrightarrow} C_{x+x^{\prime}} \text {, (binary coagulation) }
$$

and

$$
C_{x} \stackrel{b\left(x-x^{\prime}, x^{\prime}\right)}{\longrightarrow} C_{x-x^{\prime}}+C_{x^{\prime}}, \quad \text { (binary fragmentation), }
$$

where $a$ and $b$ denote the coagulation and fragmentation rates respectively, and are assumed to depend only on the size of the clusters involved in these reactions.

The dynamics of the density function $f=f(t, x) \geq 0$ of particles with mass $x \in \mathbb{R}^{+}$, at time $t \geq 0$, subject to coagulation and fragmentation phenomena is governed by the equation

$$
\frac{\partial f}{\partial t}=\mathcal{Q}_{\mathrm{c}}(f)-\mathcal{Q}_{\mathrm{f}}(f)
$$

Received by the editor March 8, 2006 and, in revised form, October 31, 2006, January 3, 2007, and March 1, 2007.

2000 Mathematics Subject Classification. Primary 65R20, 82C05.

Key words and phrases. Coagulation-fragmentation equation, finite volume method.

The second author was supported in part by ANR Grant MNEC.

(C)2007 American Mathematical Society 
where the coagulation and fragmentation terms are respectively defined by

$$
\begin{aligned}
& \mathcal{Q}_{\mathrm{c}}(f)(x)=\frac{1}{2} \int_{0}^{x} a\left(x^{\prime}, x-x^{\prime}\right) f\left(x^{\prime}\right) f\left(x-x^{\prime}\right) d x^{\prime}-\int_{0}^{\infty} a\left(x, x^{\prime}\right) f(x) f\left(x^{\prime}\right) d x^{\prime}, \\
& \mathcal{Q}_{\mathrm{f}}(f)(x)=\frac{1}{2} \int_{0}^{x} b\left(x^{\prime}, x-x^{\prime}\right) d x^{\prime} f(x)-\int_{0}^{\infty} b\left(x, x^{\prime}\right) f\left(x+x^{\prime}\right) d x^{\prime} .
\end{aligned}
$$

The coagulation coefficient, $a=a\left(x, x^{\prime}\right)$, characterizes the rate at which the coalescence of two particles with respective volumes $x$ and $x^{\prime}$ produces a particle of volume $x+x^{\prime}$, whereas the fragmentation coefficient, $b=b\left(x, x^{\prime}\right)$, represents the rate at which the fragmentation of one particle with volume $x+x^{\prime}$ produces two particles of volume $x$ and $x^{\prime}$. Both coefficients $a$ and $b$ are nonnegative symmetric functions and

$$
a, b \in L_{\mathrm{loc}}^{\infty}\left(\overline{\mathbb{R}^{+}} \times \overline{\mathbb{R}^{+}}\right) .
$$

where $\overline{\mathbb{R}}^{+}=[0, \infty)$. For symmetric kernels, we observe that during the microscopic coagulation and fragmentation processes, as depicted in equations (11)-(2), the number of particles varies with time while the total mass of particles is conserved. In terms of $f$, the total number of particles and the total mass of particles at time $t \geq 0$ are respectively given by

$$
M_{0}(t):=\int_{\mathbb{R}^{+}} f(t, x) d x, \quad M_{1}(t):=\int_{\mathbb{R}^{+}} x f(t, x) d x .
$$

It is easy to show that the total number of particles $M_{0}(t)$ is increased by coagulation events and decreased by fragmentation events, while the total mass $M_{1}(t)$ should not vary during these events.

However, when $a$ increases sufficiently rapidly compared to the fragmentation kernel $b$ for large $x, x^{\prime}$, a runaway growth takes place, producing particles with "infinite" mass in finite time which are removed from the system. The total mass is thereby not conserved, a phenomenon usually called the occurrence of gelation (see 5, 8, 9, 14, 20, 21). It is a physically relevant and mathematically challenging question to figure out whether the mass $M_{1}(t)$ of solutions to (3) is kept constant throughout time evolution.

In fact, several works in the physical literature have considered this question for the pure coagulation equation $(b=0)$ [20] and either formal arguments or explicit solutions have been provided to show that the conservation of mass holds true for $a\left(x, x^{\prime}\right)=\left(x x^{\prime}\right)^{\alpha}$ with $\alpha \in[0,1 / 2]$ and breaks down in finite time when $\alpha \in(1 / 2,1]$; see [20, 21] and the references therein.

Concerning the mathematical theory of gelation, the kernels $a\left(x, x^{\prime}\right)=x x^{\prime}$ and $b=0$ play a special role since equation (3) can be solved by the Laplace transform 23. and a mass-decreasing solution is known to exist.

Existence of weak solutions with non-increasing mass has been proven for a large class of kernels by Laurençot [15. Theorem 1.2] and completed for general kernels by Laurençot and Mischler [16, Theorem 2.3] as a limit of discrete models. Mathematical proofs of the occurrence of gelation including larger classes of coagulation rates, and also fragmentation, have been supplied recently, either by probabilistic arguments [14] (for the discrete model) or by deterministic arguments [8, 9]. Our aim is to give some numerical evidence of this phenomenon.

Writing equation (3) in a "conservative" form, as proposed in 22, 24, enables us to describe precisely the time evolution of the total mass. Also, this formulation 
is particularly well adapted to a finite volume discretization which, in turn, is expected to give a precise account of mass dissipation or conservation. Precisely, the coagulation and fragmentation terms can be written in divergence form:

$$
\left\{\begin{array}{l}
x \mathcal{Q}_{\mathrm{c}}(f)(x)=-\frac{\partial \mathcal{C}(f)}{\partial x}(x), \\
x \mathcal{Q}_{\mathrm{f}}(f)(x)=-\frac{\partial \mathcal{F}(f)}{\partial x}(x),
\end{array}\right.
$$

where

$$
\begin{aligned}
& \mathcal{C}(f)(x):=\int_{0}^{x} \int_{x-u}^{\infty} u a(u, v) f(u) f(v) d v d u, x \in \mathbb{R}^{+}, \\
& \mathcal{F}(f)(x):=\int_{0}^{x} \int_{x-u}^{\infty} u b(u, v) f(u+v) d v d u, \quad x \in \mathbb{R}^{+} .
\end{aligned}
$$

Then, the coagulation-fragmentation equation reads

$$
\left\{\begin{array}{l}
x \frac{\partial f}{\partial t}=-\frac{\partial \mathcal{C}(f)}{\partial x}+\frac{\partial \mathcal{F}(f)}{\partial x}, \quad(t, x) \in\left(\mathbb{R}^{+}\right)^{2}, \\
f(0, x)=f^{\text {in }}(x), \quad x \in \mathbb{R}^{+}
\end{array}\right.
$$

and we assume that the initial datum $f^{\text {in }}$ satisfies:

$$
f^{\text {in }} \in L^{1}\left(\mathbb{R}^{+}\right) \cap L^{1}\left(\mathbb{R}^{+}, x d x\right) \text { is a nonnegative function. }
$$

Here and below, the notation $L^{1}\left(\mathbb{R}^{+}, x d x\right)$ stands for the space of the Lebesgue measurable real-valued functions on $\mathbb{R}^{+}$which are integrable with respect to the measure $x d x$.

The main purpose of this work is to present a numerical scheme to solve (57)-(7) built upon an explicit Euler discretization with respect to the time variable $t$ and a finite volume discretization with respect to the volume variable $x$. The analysis of the so-obtained scheme allows us to prove the convergence of the discretized particle density towards a solution to the continuous problem. An error estimate on the approximation is given and the scheme is shown to give a first order accurate discretization of the coagulation-fragmentation equation.

Before describing more precisely our results, let us recall that the coagulation and fragmentation equations (5)-(7) have been the object of several studies recently.

On the one hand, the relationship between discrete and continuous models has been considered by some authors; see [25, 16] and the survey paper [5] and the references therein. In [25, the authors restrict themselves to a particular fragmentation model and use a scaling technique, whereas a rigorous setting under general assumptions on the coagulation and fragmentation coefficients has been given in 16.

Among the various approaches for the approximation of coagulation and fragmentation models, we may distinguish between deterministic and Monte Carlo methods. We refer for instance to [7, 18, for deterministic methods, [2, 6, 13. for stochastic methods, and the references therein. However, there are few results concerning the convergence analysis of numerical methods for coagulation and fragmentation models (see 17] for quasi Monte-Carlo methods). In [16, the authors obtain as a by-product of their analysis a convergence result for an explicit time discretization. However, note that the main outcome of this study is a deeper 
understanding of the link between the discrete and continuous coagulation and fragmentation equations, thanks to scaling methods, whereas the present paper is rather focused on the discretization of the continuous coagulation-fragmentation equation itself. To this aim, we use the formulation in divergence form, which is more suitable to design a finite volume scheme, and, unlike the discretization proposed in [16, this scheme is built on non-uniform meshes. Also, while reference [16] gives an analysis for unbounded domains of admissible size values, one of our goals is to assess the reliability of non-conservative truncation methods. Among other features established in this paper, these approximation methods prove to give a faithful picture of long time behaviour as well as of occurence of gelation. Indeed, the occurrence of gelation at finite time is a well-known feature of coagulation and fragmentation processes. For $a(u, v)=u v$ and $b(u, v)=0$, the behavior approaching the gelation time is studied in great detail by Menon and Pego using Laplace transform [23. Moreover, gelation has been theoretically established in [14] with a probabilistic approach and in [8, 9] with deterministic arguments. Once gelation is known to occur, a natural question is to determine the gelation time and to investigate the behavior of $f(t)$ at the gelation time, which still constitutes an open problem. In this context, numerical simulations could give some clues on how to solve this problem and, in particular, non-conservative truncation methods of approximations may prove an efficient tool to observe gelation with accuracy.

We now briefly outline the contents of the paper. In the next section, we introduce the numerical approximation of (5)-(7) and state the convergence result which we prove in Sections 3 and 4. In Section [5] we give some error estimates when the mesh is uniform. In the final section (Section 6), some numerical simulations are performed with the numerical scheme presented in Section 2, Long time behaviour and occurrence of gelation are investigated.

\section{NumeriCAL SCHEME AND MAIN RESUltS}

When designing the volume discretization of the coagulation and fragmentation terms, one is confronted with two somewhat contradictory requirements. First, the coagulation and fragmentation terms should be discretized so as to allow for the simulation of gelation phenomena for instance. But occurrence of gelation depends on the behaviour of kernels $a$ and $b$ for large values of the volume variable $x$. On the other hand, discretizing these terms makes it necessary to truncate the infinite integrals in formulae (5)-(6). But this means restricting the domain of action of kernels $a$ and $b$ to a bounded set of volumes $x$, that is, preventing coagulation to occur among particles with volume exceeding a fixed value.

The discretization we propose tries to overcome this conflict by using a nonconservative truncation method for the coagulation term. The following truncation has been introduced for the Smoluchowski coagulation equation written in the classical form (3) in 3. Here we present the equivalent truncation for the coagulationfragmentation operator written in the conservative form (5)-(6). Given a positive real $R$, let

$$
\mathcal{C}_{\mathrm{nc}}^{R}(f)(x):=\int_{0}^{x} \int_{x-u}^{R} u a(u, v) f(u) f(v) d v d u, \quad x \in(0, R) .
$$


In that case, $\mathcal{C}_{\mathrm{nc}}^{R}(f)(R) \geq 0$ so that the total mass of the solution is now nonincreasing with respect to time. This approximation is particularly well suited for reproducing the gelation phenomenon [3, 4].

For the fragmentation term, the first idea would be to give a "non-conservative" truncation as for the coagulation term, according to

$$
\mathcal{F}_{\mathrm{nc}}^{R}(f)(x):=\int_{0}^{x} \int_{x-u}^{R} u b(u, v) f(u+v) d v d u, \quad x \in(0, R),
$$

where $R$ is, as above, a constant positive parameter. Obviously, if one only considers the solution to the non-conservative fragmentation equation, it leads to a time increasing mass for the system. However, it is hard to conclude on the conservativity of the full model when including the coagulation term $-\partial_{x} \mathcal{C}_{\mathrm{nc}}^{R}$ since one should have to determine the sign of

$$
-\mathcal{C}_{\mathrm{nc}}^{R}(t, R)+\mathcal{F}_{\mathrm{nc}}^{R}(t, R), \quad t \geq 0,
$$

which is not obvious (in particular, the first term is quadratical in $f$ and depends on $a$, whereas the second one is linear in $f$ and depends on $b$ ).

Possibly the most meaningful truncation is therefore a conservative truncation on the fragmentation term (while a non-conservative truncation is performed on the coagulation part). We introduce

$$
\mathcal{F}_{\mathrm{c}}^{R}(f)(x):=\int_{0}^{x} \int_{x-u}^{R-u} u b(u, v) f(u+v) d v d u .
$$

Then, the conservative fragmentation operator satisfies exactly the conservation of total mass, so that the following equation is indeed a non-conservative coagulation and fragmentation equation:

$$
\left\{\begin{array}{l}
x \frac{\partial f_{R}}{\partial t}=-\frac{\partial \mathcal{C}_{\mathrm{nc}}^{R}\left(f_{R}\right)}{\partial x}(x)+\frac{\partial \mathcal{F}_{\mathrm{c}}^{R}\left(f_{R}\right)}{\partial x}(x), \quad(t, x) \in \mathbb{R}^{+} \times(0, R), \\
f(0, x)=f^{\text {in }}(x), \quad x \in(0, R),
\end{array}\right.
$$

since

$$
\frac{d}{d t} \int_{0}^{R} x f_{R}(t, x) d x=-\mathcal{C}_{\mathrm{nc}}^{R}\left(f_{R}\right)(t, R) \leq 0 .
$$

Convergence for large values of $R$ has been thoroughly studied in the recent past. We briefly mention some results for the coagulation equation (that is, with $b=0$ ). On the one hand, when $a\left(x, x^{\prime}\right) /\left(x x^{\prime}\right) \rightarrow 0$ as $x+x^{\prime} \rightarrow+\infty$, convergence as $R \rightarrow$ $+\infty$ of the solutions to (11) toward a solution of (5)-(7) can be proven by using the approach developed in [16]. First, we observe that the previous growth assumption on $a\left(x, x^{\prime}\right)$ does not exclude coagulation coefficients for which the occurrence of gelation takes place. Second, when gelation does not take place, it can be shown that the solutions to (11) converge toward a solution to (5) -(7) satisfying $M_{1}(t)=M_{1}(0)$ for $t \geq 0$ (we refer to [11] for a rigorous proof on the coagulation equation). On the other hand, the convergence of the nonconservative approximation (11) to the solution of (5)-(77) is valid when $a\left(x, x^{\prime}\right) \sim x x^{\prime}$ for large $x, x^{\prime}$ [15]. Therefore, this approximation is well-suited for the description of both gelation and mass conservation, despite the qualitatively important gap between these regimes. 
Let us mention that these results and analysis adapt easily to the coagulationfragmentation equation but under different assumptions on the kernels (we refer to [8] and [9] for a precise description of kernels).

Since the convergence of solutions to (11) towards solutions of (15)-(7) is well established in rather general situations, this paper will only focus on the convergence of a sequence built on a numerical scheme towards a solution to the equation (11) when the truncature $R$ is fixed. The works we have just mentioned fill in the gap to get a convergence result to solutions to the original problem. In the remainder of the paper, for the sake of clarity, we drop the subscript $R$ and write $f$ instead of $f_{R}$ for a solution of equation (11). Parameter $R$ being fixed, this should raise no confusion.

Now, we turn to the discretization of equation (11). Having reduced the computation to a bounded interval, the second step is to introduce the time and volume discretizations. To this end, let $h \in(0,1), I^{h}$ a large integer, and denote by $\left(x_{i-1 / 2}\right)_{i \in\left\{0, \ldots, I^{h}\right\}}$ a mesh of $(0, R)$, where

$$
x_{-1 / 2}=0, \quad x_{i}=\left(x_{i-1 / 2}+x_{i+1 / 2}\right) / 2, \quad \Delta x_{i}=x_{i+1 / 2}-x_{i-1 / 2} \leq h,
$$

and $\Lambda_{i}^{h}=\left[x_{i-1 / 2}, x_{i+1 / 2}\right)$ for $i \geq 0$. Moreover, given two integers $i$ and $j$ such that $x_{i+1 / 2}-x_{j} \geq 0$, we introduce integer $\gamma_{i, j} \in\left\{0, \ldots, I^{h}\right\}$ such that

$$
x_{i+1 / 2}-x_{j} \in \Lambda_{\gamma_{i, j}}^{h} .
$$

In the general case of a non-uniform mesh, we denote by $\delta_{h}=\min \Delta x_{i}$ and assume that there exists a positive constant (independent of the mesh) ${ }^{i} K$ such that

$$
\frac{h}{\delta_{h}} \leq K
$$

or, if the mesh has to be excessively refined in some regions (usually close to the origin), we assume that the mesh is increasing, that is,

$$
\Delta x_{i} \leq \Delta x_{i+1}, \quad \forall i \in\left\{0, \ldots, I^{h}-1\right\} .
$$

Remark 2.1. In the case of a uniform mesh (that is when $\Delta x_{i}=h$ for all $i$ ), it holds that

$$
x_{i-\frac{1}{2}}=i h, \quad \gamma_{i, j}=i-j .
$$

In particular, whenever $x_{j-\frac{1}{2}}<x<x_{j+\frac{1}{2}}$ and $j<i$, we have

$$
x_{i+\frac{1}{2}}-x \in \Lambda_{i-j}^{h} \text {. }
$$

Let $\Delta t$ denote the time step and let $N$ be a large integer such that $N \Delta t=T$, where $[0, T]$ is the time domain on which the equation is studied. We also define the time interval $\tau_{n}=\left[t_{n}, t_{n+1}\right)$, with $t^{n}=n \Delta t, n \geq 0$.

The discretization of the coagulation and fragmentation kernels will be detailed at the end of this section. For the time being, we formally set

$$
\begin{aligned}
a(u, v) & \approx a^{h}(u, v)=a_{i, j}, \\
b(u, w-u) & \approx b^{h}(u, w-u)=b_{i, k}
\end{aligned}
$$

for $u$ in $\Lambda_{i}^{h}, v$ in $\Lambda_{j}^{h}$ and $w$ in $\Lambda_{k}^{h}$, such that $i, j \in\left\{0, \ldots, I^{h}\right\}$ and $k \in\left\{i+1, \ldots, I^{h}\right\}$ and assume that this defines a suitable approximation of the kernels. 
Let us now introduce the numerical scheme itself. For each integer $i \in\left\{0, \ldots, I^{h}\right\}$ and each $n \in\{0, \ldots, N-1\}$, we define the approximation of $f(t, x)$ for $t \in \tau_{n}$ and $x \in \Lambda_{i}^{h}$ as $f_{i}^{n}$.

The sequence $\left(f_{i}^{n}\right)_{i, n}$ is defined recursively by the following discretization of the coagulation-fragmentation equation: for $n \in\{1, \ldots, N-1\}, i \in\left\{0, \ldots, I^{h}\right\}$, we set

$$
\Delta x_{i} x_{i}\left(f_{i}^{n+1}-f_{i}^{n}\right)=-\Delta t\left(\mathcal{C}_{i+1 / 2}^{n}-\mathcal{C}_{i-1 / 2}^{n}\right)+\Delta t\left(\mathcal{F}_{i+1 / 2}^{n}-\mathcal{F}_{i-1 / 2}^{n}\right),
$$

where the fluxes $\mathcal{C}_{i+1 / 2}^{n}$ and $\mathcal{F}_{i+1 / 2}^{n}$ are given by

$$
\begin{aligned}
\mathcal{C}_{i+1 / 2}^{n} & =\sum_{j=0}^{i} \sum_{k=\gamma_{i, j}}^{I^{h}} \Delta x_{j} \Delta x_{k} x_{j} a_{j, k} f_{j}^{n} f_{k}^{n}, \\
\mathcal{F}_{i+1 / 2}^{n} & =\sum_{j=0}^{i} \sum_{k=i+1}^{I^{h}} \Delta x_{j} \Delta x_{k} x_{j} b_{j, k} f_{k}^{n},
\end{aligned}
$$

and the initial datum is approached by

$$
f_{i}^{\text {in }}=\frac{1}{\Delta x_{i}} \int_{\Lambda_{i}^{h}} f^{\text {in }}(x) d x, \quad i \in\left\{0, \ldots, I^{h}\right\},
$$

whereas the fluxes at the boundary are

$$
\mathcal{C}_{-1 / 2}^{n}=\mathcal{F}_{-1 / 2}^{n}=\mathcal{F}_{I^{h}+1 / 2}^{n}=0, \quad n \in\{0, \ldots, N-1\} .
$$

This discretization obviously relies on an explicit Euler time discretization and a finite volume approach for the volume variable (see, e.g. [10, 19]). This will be even clearer when the discretization of $a$ and $b$ are given.

We denote by $\chi_{\mathcal{A}}$ the characteristic function of a set $\mathcal{A}$. The following function $f^{h}$ defined on $[0, T] \times[0, R]$ will be useful in the following:

$$
f^{h}(t, x)=\sum_{n=0}^{N-1} \sum_{i=0}^{I^{h}} f_{i}^{n} \chi_{\tau_{n}}(t) \chi_{\Lambda_{i}^{h}}(x) .
$$

Note that this function depends on the time and volume steps and that

$$
f^{h}(0, \cdot)=\sum_{i=0}^{I^{h}} \chi_{\Lambda_{i}^{h}}(\cdot) f_{i}^{\text {in }}
$$

converges strongly to $f^{\text {in }}$ in $L^{1}(0, R)$ as $h$ goes to 0 .

We may now state our main result.

Theorem 2.2. Assume that the coagulation and fragmentation kernels satisfy (4) and $f^{i n}$ satisfies (8). Moreover, suppose that the volume mesh used in the numerical scheme is regular in the sense of assumptions (12) or (13) and that the time step satisfies that there exists a positive constant $\theta$ such that

$$
\max (2, K+1) C_{T, R} \Delta t \leq \theta<1
$$

where $K$ is given by (12) and

$$
C_{T, R}:=\|a\|_{L^{\infty}}\left\|f^{i n}\right\|_{L^{1}} e^{R\|b\|_{L^{\infty} T}}+R\|b\|_{L^{\infty}} .
$$

Then, up to the extraction of a subsequence,

$$
f^{h} \longrightarrow f \text { in } L^{\infty}\left(0, T ; L^{1}(0, R)\right)
$$


where $f$ is the weak solution to (11) on $[0, T]$ with initial datum $f^{\text {in }}$. More precisely, $f$ is a nonnegative function satisfying

$$
\begin{aligned}
& \int_{0}^{T} \int_{0}^{R} x f(t, x) \frac{\partial \varphi}{\partial t}(t, x)+\left[\mathcal{C}_{n c}^{R}(t, x)-\mathcal{F}_{c}^{R}(t, x)\right] \frac{\partial \varphi}{\partial x}(t, x) d x d t \\
& +\int_{0}^{R} x f^{i n}(x) \varphi(0, x) d x-\int_{0}^{T} \mathcal{C}_{n c}^{R}(t, R) \varphi(t, R) d t=0,
\end{aligned}
$$

for all continuously differentiable functions $\varphi$ compactly supported in $[0, T) \times[0, R]$.

Moreover, when the mesh is uniform $\Delta x_{i}=h$, for all $i \in\left\{0, \ldots, I^{h}\right\}$, we get the following error estimate.

Theorem 2.3. Assume that the coagulation and fragmentation kernels satisfy

$$
a, b \in W_{l o c}^{1, \infty}\left(\mathbb{R}^{+} \times \mathbb{R}^{+}\right) .
$$

We also assume that $f^{\text {in }}$ satisfies

$$
f^{i n} \in W_{l o c}^{1, \infty}\left(\mathbb{R}^{+}\right) .
$$

We consider a uniform volume mesh and require time step $\Delta t$ to satisfy condition (22). Then, the following error estimate holds:

$$
\left\|f^{h}-f\right\|_{L^{1}} \leq C(T, R)(h+\Delta t),
$$

where $f$ is the weak solution to (11) on $[0, T]$ with initial datum $f^{i n}$.

This implies the uniqueness of the limit, and, consequently, that the whole sequence $f^{h}$ converges under these assumptions.

Of course, these results depend on the definition of a correct approximation of the coagulation and fragmentation kernels. Equations (14) and (15) are now given a precise meaning.

Unless otherwise specified, in the following kernels $a$ and $b$ are taken as in (4). On the one hand, the kernel $a$ is approached by a finite volume approximation $a^{h}(u, v)$ on each space cell: for all $(u, v) \in[0, R] \times[0, R]$,

$$
a^{h}(u, v)=\sum_{i=0}^{I^{h}} \sum_{j=0}^{I^{h}} a_{i, j} \chi_{\Lambda_{i}^{h}}(u) \chi_{\Lambda_{j}^{h}}(v),
$$

where $\chi_{\mathcal{A}}$ denotes the characteristic function of set $\mathcal{A}$ and

$$
a_{i, j}=\frac{1}{\Delta x_{i} \Delta x_{j}} \int_{\Lambda_{i}^{h} \times \Lambda_{j}^{h}} a(x, y) d x d y .
$$

This approximation method is well-known and yields strong convergence in the space of integrable functions provided the kernel $a$ is in $L^{1}((0, R) \times(0, R))$, which is the case here according to (4),

$$
\left\|a^{h}-a\right\|_{L^{1}} \rightarrow 0, \quad \text { as } h \rightarrow 0 .
$$

The fragmentation kernel $b$ is discretized in a different way. Indeed, we first notice that $b$ need not be defined on the whole square $[0, R] \times[0, R]$ and that the kernel defined on the compact set

$$
\mathcal{D}_{b}:=\{(u, v) \in[0, R] \times[0, R] ; \quad 0 \leq u+v \leq R\}
$$


can be used to compute the fragmentation term $\mathcal{F}_{\mathrm{c}}^{R}$ as given in (10). Therefore, all we need is to give an approximation $b^{h}$ of the kernel $b$ on the compact set $\mathcal{D}_{b}$. First, we define the following finite volume approximation of $b$, for all $(u, v) \in \mathcal{D}_{b}$,

$$
\tilde{b}^{h}(u, v)=\sum_{i=0}^{I^{h}} \sum_{j=i+1}^{I^{h}} \tilde{b}_{i, j} \chi_{\Lambda_{i}^{h}}(u) \chi_{\Lambda_{j}^{h}}(v+u)
$$

with

$$
\tilde{b}_{i, j}=\frac{1}{\Delta x_{i} \Delta x_{j}} \int_{\Lambda_{i}^{h} \times \Lambda_{j}^{h}} b(x, y-x) d y d x, \quad 0 \leq i<j \leq I^{h} .
$$

This sequence is particularly well suited to approach $b(u, v-u)$ since, for all $(u, v) \in$ $[0, R] \times[0, R]$ such that $0 \leq u \leq v \leq R$,

$$
\tilde{b}^{h}(u, v-u)=\sum_{i=0}^{I^{h}} \sum_{j=i+1}^{I^{h}} \tilde{b}_{i, j} \chi_{\Lambda_{i}^{h}}(u) \chi_{\Lambda_{j}^{h}}(v)
$$

is obviously a finite volume approximation of $b(u, v-u)$. Then, since $b$ is symmetric, one would expect that a good approximation $b^{h}$ of $b$ should satisfy

$$
b^{h}(u, v)=b^{h}(v, u)
$$

which would translate on the sequence $b_{i, j}$ as

$$
b_{i, j}=b_{j-i, j} .
$$

However, this is not true in general and one can prove that, in the case of a more regular kernel $b$ satisfying condition (25) and for a uniform mesh, equality (30) holds true only up to first order terms in $h$ when $b_{i, j}=\tilde{b}_{i, j}$.

This motivates the following definition:

Definition 2.4. We define the approximate kernel $b^{h}$ such that

( $i$ ) if the fragmentation $b$ satisfies (4), then an approximation of $b$ is defined by

$$
b^{h}(u, v)=\tilde{b}^{h}(u, v), \quad \forall(u, v) \in \mathcal{D}_{b},
$$

where $\tilde{b}^{h}$ is given by (28) and (29);

(ii) if the fragmentation kernel $b$ satisfies (25) and if the mesh is taken uniformly, then one introduces the following approximation of $b$,

$$
b^{h}(u, v)=\sum_{i=0}^{I^{h}} \sum_{j=i+1}^{I^{h}} b_{i, j} \chi_{\Lambda_{i}^{h}}(u) \chi_{\Lambda_{j}^{h}}(v+u), \quad \forall(u, v) \in \mathcal{D}_{b},
$$

where

$$
b_{i, j}= \begin{cases}\frac{1}{2}\left(\tilde{b}_{i, j}+\tilde{b}_{j-i, j}\right), & i \in\left\{1, \ldots, \mathcal{I}^{h}\right\}, j \in\left\{i+1, \ldots, I^{h}\right\}, \\ \tilde{b}_{0, j}, & i=0, j \in\left\{1, \ldots, I^{h}\right\} .\end{cases}
$$

It will be useful to write, conventionally,

$$
b_{0,0}=0 .
$$

Properties of these approximations are summarized in the following lemma.

Lemma 2.5. The approximate kernel $b^{h}$ given either by (31) or by (32) satisfies the following convergence properties: 
(i) Let $b$ satisfy (44), then equation (31) defines an approximation of $b$ which converges strongly in the $L^{1}$ topology

$$
\left\|b^{h}-b\right\|_{L^{1}\left(\mathcal{D}_{b}\right)} \rightarrow 0, \quad \text { as } h \rightarrow 0 .
$$

(ii) Let b satisfy (25), and take a uniform mesh. Then the approximation of $b$ given by equation (32) converges in the strong topology of $L^{1}$,

$$
\left\|b^{h}-b\right\|_{L^{1}\left(\mathcal{D}_{b}\right)} \rightarrow 0, \quad \text { as } h \rightarrow 0,
$$

and the sequence $b_{i, j}$ defined in (33) satisfies

$$
b_{i, j}=b_{j-i, j}, \quad i \in\left\{1, \ldots, \mathcal{I}^{h}\right\}, j \in\left\{i+1, \ldots, I^{h}\right\} .
$$

Proof. Strong convergence for the approximation (31) is classical: the proof is close to the convergence proof of the finite volume approximation $a^{h}$.

On the other hand, when the kernel $b$ satisfies (25), we can perform a Taylor expansion of $b$ and easily prove that

$$
\left|\tilde{b}_{i, j}-\tilde{b}_{j-i, j}\right| \leq\|b\|_{W^{1, \infty}} h,
$$

which means that up to a first order term with respect to $h$, the approximation (32) is equal to the approximation (31). Therefore, strong convergence for (31) implies strong convergence for (32) since the first order term in $h$ vanishes asymptotically.

Remark 2.6. It is worth mentioning that property (35) is used only in the proof of Theorem 2.3 to estimate the error between the numerical scheme and the actual solution of (11). In this case, we use approximation (32), take a uniform mesh and assume regularity (25) for $b$. In contrast, Theorem 2.2 can be proven without appealing to symmetry property (35) and, therefore, can be established under the weaker assumption (4), using approximation (31). Of course, it also holds true under assumption (25) and with approximation (32).

In the convergence analysis of the numerical scheme, it will be useful to consider pointwise convergence for the coagulation and fragmentation kernels. These convergences hold true up to the extraction of subsequences. Namely, there exists a subfamily of the family $\mathbb{R}_{*}^{+}$of indices $h$ such that, for almost every $(u, v) \in[0, R] \times[0, R]$ and almost every $(x, y) \in \mathcal{D}_{b}$,

$$
a^{h}(u, v) \rightarrow a(u, v), \quad b^{h}(x, y) \rightarrow b(x, y)
$$

as $h$ goes to 0 . In the remainder of this paper, all sequences will be indexed on this subfamily of indices, so that these almost everywhere convergences can be used.

\section{A PRIORI ESTIMATES}

In this section, our goal is to prove that the sequence of functions $\left(f^{h}\right)_{h}$ converges in some sense to a function $f$ as $h$ and $\Delta t$ go to 0 . First, we prove that the solution $f^{h}$ to the scheme (16)-(19) enjoys properties similar to those of function $f$ given by (11) which we gather in Proposition 3.1 below. Next, we prove the weak convergence of $f^{h}$ to a function $f$ in $L^{1}(0, R)$.

The midpoint approximation of a point $x$ is denoted by $X^{h}(x)$, i.e. $X^{h}(x)=x_{i}$ for $x \in \Lambda_{i}^{h}$; see Section 4 for further details. 
Proposition 3.1. Assume the time step satisfies (22). Then, the distribution function $f^{h}$ is a nonnegative function such that

$$
\int_{0}^{R} X^{h}(x) f^{h}(t, x) d x \leq \int_{0}^{R} X^{h}(x) f^{h}(s, x) d x, \quad 0 \leq s \leq t \leq T
$$

and, for all $t \in[0, T]$,

$$
\int_{0}^{R} f^{h}(t, x) d x \leq\left\|f^{\mathrm{in}}\right\|_{L^{1}} e^{R\|b\|_{L} \infty t} .
$$

Proof. We proceed by induction and first notice that $f^{h}(0)$ is nonnegative and belongs to $L^{1}(0, R)$. Assume next that the function $f^{h}\left(t^{n}\right)$ is nonnegative and

$$
\int_{0}^{R} f^{h}\left(t^{n}, x\right) d x \leq\left\|f^{\mathrm{in}}\right\|_{L^{1}} e^{R\|b\|_{L^{\infty}} t^{n}}
$$

We start by proving that $f^{h}\left(t^{n+1}\right)$ is nonnegative, and first take $i=0$ since it involves boundary conditions,

$$
\begin{aligned}
x_{0} f_{0}^{n+1} & =x_{0} f_{0}^{n}-\frac{\Delta t}{\Delta x_{0}} \mathcal{C}_{1 / 2}^{n}+\frac{\Delta t}{\Delta x_{0}} \mathcal{F}_{1 / 2}^{n}, \\
& \geq\left(1-\Delta t \sum_{k=0}^{I^{h}} \Delta x_{k} a_{0, k} f_{k}^{n}\right) x_{0} f_{0}^{n} .
\end{aligned}
$$

Then, using condition (22) on the time step with (37), we conclude to the nonnegativity of $f_{0}^{n+1}$. For $i \geq 1$, we have

$$
x_{i} f_{i}^{n+1}=x_{i} f_{i}^{n}-\frac{\Delta t}{\Delta x_{i}}\left(\mathcal{C}_{i+1 / 2}^{n}-\mathcal{C}_{i-1 / 2}^{n}\right)+\frac{\Delta t}{\Delta x_{i}}\left(\mathcal{F}_{i+1 / 2}^{n}-\mathcal{F}_{i-1 / 2}^{n}\right) .
$$

On the one hand, from the nonnegativity of $f^{h}\left(t^{n}\right)$, we show that

$$
\begin{aligned}
-\frac{\mathcal{C}_{i+1 / 2}^{n}-\mathcal{C}_{i-1 / 2}^{n}}{\Delta x_{i}}= & -x_{i} f_{i}^{n} \sum_{k=\gamma_{i, i}}^{I^{h}} \Delta x_{k} a_{i, k} f_{k}^{n} \\
& +\sum_{j=0}^{i-1} \sum_{k=\gamma_{i-1, j}}^{\gamma_{i, j}-1} \frac{\Delta x_{k}}{\Delta x_{i}} f_{k}^{n} \Delta x_{j} a_{j, k} x_{j} f_{j}^{n} \\
\geq & -\left(\sum_{k=0}^{I^{h}} \Delta x_{k} a_{i, k} f_{k}^{n}\right) x_{i} f_{i}^{n} .
\end{aligned}
$$

On the other hand, still using the nonnegativity of $f^{h}\left(t^{n}\right)$, we get for the discrete fragmentation operator

$$
\begin{aligned}
\frac{\mathcal{F}_{i+1 / 2}^{n}-\mathcal{F}_{i-1 / 2}^{n}}{\Delta x_{i}} & =\sum_{k=i+1}^{I^{h}} \Delta x_{k} x_{i} b_{i, k} f_{k}^{n}-\sum_{j=0}^{i-1} \Delta x_{j} b_{j, i} x_{j} f_{i}^{n} \\
& \geq-\left(\sum_{k=0}^{I^{h}} \Delta x_{k} b_{k, i}\right) x_{i} f_{i}^{n} .
\end{aligned}
$$


Then gathering the two inequalities (38) and (39), we get

$$
x_{i} f_{i}^{n+1} \geq\left(1-\Delta t\left(\sum_{k=0}^{I^{h}} \Delta x_{k} a_{i, k} f_{k}^{n}+\sum_{k=0}^{I^{h}} \Delta x_{k} b_{k, i}\right)\right) x_{i} f_{i}^{n} .
$$

Using condition (22) on the time step and the $L^{1}$-estimate (37) on $f^{h}\left(t^{n}\right)$, we finally prove that $f^{h}\left(t^{n+1}\right)$ is nonnegative.

Next, the time monotonicity of the total mass with respect to time follows at once from the nonnegativity of $f^{h}$ by summing (16) with respect to $i$

$$
\sum_{i=0}^{I^{h}} \Delta x_{i} x_{i} f_{i}^{n+1} \leq \sum_{i=0}^{I^{h}} \Delta x_{i} x_{i} f_{i}^{n}-\Delta t \mathcal{C}_{I^{h}+1 / 2}^{n} \leq \sum_{i=0}^{I^{h}} \Delta x_{i} x_{i} f_{i}^{n} .
$$

Now, let us prove that $f^{h}\left(t^{n+1}\right)$ enjoys a similar estimate as (37). It follows from (16) that

$\sum_{i=0}^{I^{h}} \Delta x_{i} f_{i}^{n+1}=\sum_{i=0}^{I^{h}} \Delta x_{i} f_{i}^{n}-\Delta t \sum_{i=0}^{I^{h}} \frac{\mathcal{C}_{i+1 / 2}^{n}-\mathcal{C}_{i-1 / 2}^{n}}{x_{i}}+\Delta t \sum_{i=0}^{I^{h}} \frac{\mathcal{F}_{i+1 / 2}^{n}-\mathcal{F}_{i-1 / 2}^{n}}{x_{i}}$.

Of course the coagulation term decreases the number of particles $\left(\mathcal{C}_{i+1 / 2} \geq 0\right.$, for all $i$ )

$$
-\sum_{i=0}^{I^{h}} \frac{\mathcal{C}_{i+1 / 2}^{n}-\mathcal{C}_{i-1 / 2}^{n}}{x_{i}} \leq-\sum_{i=0}^{I^{h}} \mathcal{C}_{i+1 / 2}^{n}\left(\frac{1}{x_{i}}-\frac{1}{x_{i+1}}\right) \leq 0 .
$$

For the fragmentation term, we observe that

$$
\sum_{i=0}^{I^{h}} \frac{\mathcal{F}_{i+1 / 2}^{n}-\mathcal{F}_{i-1 / 2}^{n}}{x_{i}} \leq \sum_{i=0}^{I^{h}} \Delta x_{i} \sum_{k=i+1}^{I^{h}} \Delta x_{k} b_{i, k} f_{k}^{n}
$$

and using the assumption (4) on the kernel $b$, we finally get

$$
\sum_{i=0}^{I^{h}} \Delta x_{i} f_{i}^{n+1} \leq\left(1+R\|b\|_{L^{\infty}} \Delta t\right) \sum_{i=0}^{I^{h}} \Delta x_{i} f_{i}^{n} .
$$

Thus, using estimate (37) at step $n$,

$$
\sum_{i=0}^{I^{h}} \Delta x_{i} f_{i}^{n+1} \leq\left\|f^{\mathrm{in}}\right\|_{L^{1}} e^{R\|b\|_{L^{\infty}} t^{n+1}} .
$$

We also remark that $f^{h}(0, \cdot)$ is an approximation of $f^{\text {in }}$, with strong convergence in $L^{1}(0, R)$. Moreover, the initial datum $f^{\text {in }}$ is in $L^{1}(0, R)$, hence, by the La ValléePoussin theorem, there exists a nonnegative and convex function $\Phi$ continuously differentiable on $\mathbb{R}^{+}$with $\Phi(0)=0, \Phi^{\prime}(0)=1$, such that $\Phi^{\prime}$ is concave,

$$
\frac{\Phi(r)}{r} \longrightarrow+\infty, \text { as } r \rightarrow \infty
$$

and

$$
\int_{0}^{R} \Phi\left(f^{\text {in }}\right)(x) d x<+\infty .
$$

Let us now recall an inequality on convex functions. 
Lemma 3.2. Let $\Phi \in C^{1}\left(\mathbb{R}^{+}\right)$be convex such that $\Phi^{\prime}$ is concave, $\Phi(0)=0, \Phi^{\prime}(0)=$ 1 , and $\Phi(r) / r \rightarrow+\infty$ as $r \rightarrow+\infty$. Then, for all $(x, y)$ in $\mathbb{R}^{+} \times \mathbb{R}^{+}$,

$$
x \Phi^{\prime}(y) \leq \Phi(x)+\Phi(y) .
$$

Next, the following result holds.

Proposition 3.3. Let $f^{i n} \in L^{1}(0, R)$ be nonnegative, and let $f^{h}$ be defined for all $h$ and $\Delta t$ by (16)-(21) where $\Delta t$ satisfies (22). Then the family $\left(f^{h}\right)_{(h, \Delta t)}$ is weakly relatively sequentially compact in $L^{1}((0, T) \times(0, R))$.

Proof. Based on estimate (40), one can prove a similar estimate on the function $f^{h}$, uniformly in $h$. First, the integral of $\Phi\left(f^{h}\right)$ is clearly related to the sequence $f_{i}^{n}$ through

$$
\int_{0}^{T} \int_{0}^{R} \Phi\left(f^{h}(t, x)\right) d x d t=\sum_{n=0}^{N-1} \sum_{i=0}^{I^{h}} \Delta t \Delta x_{i} \Phi\left(f_{i}^{n}\right) .
$$

From the discrete equation (16), together with the convexity of the function $\Phi$ and the nonnegativity of $\Phi^{\prime}$, it follows that

$$
\begin{aligned}
& \sum_{i=0}^{I^{h}} \Delta x_{i}\left[\Phi\left(f_{i}^{n+1}\right)-\Phi\left(f_{i}^{n}\right)\right] \\
& \leq \sum_{i=0}^{I^{h}} \Delta x_{i}\left(f_{i}^{n+1}-f_{i}^{n}\right) \Phi^{\prime}\left(f_{i}^{n+1}\right) \\
& \leq\|a\|_{L^{\infty}} \Delta t \sum_{i=0}^{I^{h}} \sum_{j=0}^{i-1} \Delta x_{j} f_{j}^{n} \sum_{k=\gamma_{i-1, j}}^{\gamma_{i, j}-1} \Delta x_{k} f_{k}^{n} \Phi^{\prime}\left(f_{i}^{n+1}\right) \\
& \quad+\|b\|_{L^{\infty}} \Delta t \sum_{i=0}^{I^{h}} \sum_{k=i+1}^{I^{h}} \Delta x_{i} \Delta x_{k} f_{k}^{n} \Phi^{\prime}\left(f_{i}^{n+1}\right),
\end{aligned}
$$

where we have used assumption (4) and the fact that $x_{j} / x_{i} \leq 1$ whenever $j \leq i$. Then the convexity of $\Phi$ together with (41), entails

$$
\begin{aligned}
& \|a\|_{L^{\infty}} \Delta t \sum_{i=0}^{I^{h}} \sum_{j=0}^{i-1} \Delta x_{j} f_{j}^{n} \sum_{k=\gamma_{i-1, j}}^{\gamma_{i, j}-1} \Delta x_{k} f_{k}^{n} \Phi^{\prime}\left(f_{i}^{n+1}\right) \\
& \leq\|a\|_{L^{\infty}} \Delta t \sum_{i=0}^{I^{h}} \sum_{j=0}^{i-1} \Delta x_{j} f_{j}^{n} \sum_{k=\gamma_{i-1, j}}^{\gamma_{i, j}-1} \Delta x_{k}\left[\Phi\left(f_{k}^{n}\right)+\Phi\left(f_{i}^{n+1}\right)\right] \\
& =\|a\|_{L^{\infty}} \Delta t \sum_{j=0}^{I^{h}} \Delta x_{j} f_{j}^{n} \sum_{i=j+1}^{I^{h}} \sum_{k=\gamma_{i-1, j}}^{\gamma_{i, j}-1} \Delta x_{k}\left[\Phi\left(f_{k}^{n}\right)+\Phi\left(f_{i}^{n+1}\right)\right],
\end{aligned}
$$


and still applying (41) to the fragmentation term, it yields

$$
\begin{aligned}
& \sum_{i=0}^{I^{h}} \Delta x_{i}\left[\Phi\left(f_{i}^{n+1}\right)-\Phi\left(f_{i}^{n}\right)\right] \\
& \quad \leq\|a\|_{L^{\infty}} \Delta t \sum_{j=0}^{I^{h}} \Delta x_{j} f_{j}^{n} \sum_{i=j+1}^{I^{h}} \sum_{k=\gamma_{i-1, j}}^{\gamma_{i, j}-1} \Delta x_{k}\left[\Phi\left(f_{k}^{n}\right)+\Phi\left(f_{i}^{n+1}\right)\right] \\
& \quad+\|b\|_{L^{\infty}} \Delta t \sum_{i=0}^{I^{h}} \Delta x_{i} \sum_{k=i+1}^{I^{h}} \Delta x_{k}\left[\Phi\left(f_{k}^{n}\right)+\Phi\left(f_{i}^{n+1}\right)\right] .
\end{aligned}
$$

On the one hand, we have

$$
\sum_{i=j+1}^{I^{h}} \sum_{k=\gamma_{i-1, j}}^{\gamma_{i, j}-1} \Delta x_{k} \Phi\left(f_{k}^{n}\right)=\sum_{k=\gamma_{j, j}}^{\gamma_{I^{h}, j}-1} \Delta x_{k} \Phi\left(f_{k}^{n}\right) \leq \sum_{k=0}^{I^{h}} \Delta x_{k} \Phi\left(f_{k}^{n}\right) .
$$

On the other hand, observing that

$$
\sum_{k=\gamma_{i-1, j}}^{\gamma_{i, j}-1} \Delta x_{k}=x_{\gamma_{i, j}-1 / 2}-x_{\gamma_{i-1, j}-1 / 2}
$$

and since $x_{\gamma_{i, j}-1 / 2}$ and $x_{\gamma_{i-1, j}-1 / 2}$ are defined as left point approximations of $x_{i+1 / 2}-x_{j}$ and $x_{i-1 / 2}-x_{j}$ respectively, it gives

$$
\begin{gathered}
x_{\gamma_{i, j}-1 / 2}-x_{\gamma_{i-1, j}-1 / 2}=\left(x_{\gamma_{i, j}-1 / 2}-x_{i+1 / 2}\right)-\left(x_{\gamma_{i-1, j}-1 / 2}-x_{i-1 / 2}\right)+\Delta x_{i} \\
=\left(x_{i-1 / 2}-x_{j}\right)-x_{\gamma_{i-1, j}-1 / 2}-\left(\left(x_{i+1 / 2}-x_{j}\right)-x_{\gamma_{i, j}-1 / 2}\right)+\Delta x_{i} \\
\leq\left(x_{i-1 / 2}-x_{j}\right)-x_{\gamma_{i-1, j}-1 / 2}+\Delta x_{i} .
\end{gathered}
$$

Either assumption (12) or assumption (13) holds, and we get, accordingly,

$$
\left(x_{i-1 / 2}-x_{j}\right)-x_{\gamma_{i-1, j}-1 / 2} \leq h
$$

or

$$
\left(x_{i-1 / 2}-x_{j}\right)-x_{\gamma_{i-1, j}-1 / 2} \leq \Delta x_{i} .
$$

The latter inequality is a consequence of the fact that the mesh is increasing. In the former case, however, the regularity of the mesh is such that, by (12), one has

$$
h \leq K \Delta x_{i} .
$$

Finally, both cases yield

$$
x_{\gamma_{i, j}-1 / 2}-x_{\gamma_{i-1, j}-1 / 2} \leq M \Delta x_{i}
$$

with $M=1+K$ or $M=2$. Therefore,

$$
\sum_{i=j+1}^{I^{h}} \sum_{k=\gamma_{i-1, j}}^{\gamma_{i, j}-1} \Delta x_{k} \Phi\left(f_{i}^{n+1}\right) \leq M \sum_{i=0}^{I^{h}} \Delta x_{i} \Phi\left(f_{i}^{n+1}\right) .
$$


Consequently, this result and inequality (42) lead to

$$
\begin{aligned}
& \sum_{i=0}^{I^{h}} \Delta x_{i}\left[\Phi\left(f_{i}^{n+1}\right)-\Phi\left(f_{i}^{n}\right)\right] \\
& \quad \leq \Delta t\|a\|_{L^{\infty}}\left(\sum_{j=0}^{I^{h}} \Delta x_{j} f_{j}^{n}\right) \sum_{i=0}^{I^{h}} \Delta x_{i}\left[M \Phi\left(f_{i}^{n+1}\right)+\Phi\left(f_{i}^{n}\right)\right],
\end{aligned}
$$

which entails, as $\|a\|_{L^{\infty}} \sum \Delta x_{j} f_{j}^{n}$ is bounded by $C_{T, R}$ according to (23) and (36),

$$
\begin{aligned}
\sum_{i=0}^{I^{h}} \Delta x_{i}\left[\Phi\left(f_{i}^{n+1}\right)-\Phi\left(f_{i}^{n}\right)\right] & \leq \Delta t M C_{T, R} \sum_{i=0}^{I^{h}} \Delta x_{i} \Phi\left(f_{i}^{n+1}\right) \\
& +\Delta t C_{T, R} \sum_{i=0}^{I^{h}} \Delta x_{i} \Phi\left(f_{i}^{n}\right) .
\end{aligned}
$$

Finally, it appears that if the time step satisfies (22), a discrete version of Gronwall's lemma gives

$$
\int_{0}^{R} \Phi\left(f^{h}(t, x)\right) d x \leq e^{\frac{C_{T, R}(1+M)}{1-\theta} t} \int_{0}^{R} \Phi\left(f^{\text {in }}(x)\right) d x, \quad \forall t \in[0, T)
$$

and this estimate allows us to conclude to the compactness of the sequence $\left(f^{h}\right)_{h}$ due to the La Vallée-Poussin theorem. Indeed, the exponent is uniformly bounded with respect to $h$ and $\Delta t$ as long as (22) holds true.

Remark 3.4. Proposition 3.3 implies that there exist a function $f$ in $L^{1}((0, T) \times$ $(0, R))$ and a subsequence of $\left(f^{h}\right)_{h}$ such that $f^{h} \rightarrow f$ as $h \rightarrow 0$. By a diagonal procedure, one can extract subsequences of $\left(f^{h}\right)_{h},\left(a^{h}\right)_{h}$ and $\left(b^{h}\right)_{h}$ such that

$$
f^{h} \rightarrow f, \quad \text { in the weak topology of } L^{1}((0, T) \times(0, R)), \quad \text { as } \max \{h, \Delta t\} \rightarrow 0
$$

and

$$
\begin{aligned}
& a^{h}(u, v) \rightarrow a(u, v), \quad \text { for almost every }(u, v) \in(0, R) \times(0, R), \quad \text { as } h \rightarrow 0, \\
& b^{h}(u, v) \rightarrow b(u, v), \quad \text { for almost every }(u, v) \in \mathcal{D}_{b}, \quad \text { as } h \rightarrow 0 .
\end{aligned}
$$

Next, these diagonally extracted subsequences are considered implicitly, unless otherwise specified.

Thus, Proposition 3.3 gives enough information to study the asymptotic behaviour of all terms in equation (16). However, the following lemma recalls a classical tool that will be needed afterwards.

Lemma 3.5. Let $\Omega$ be an open subset of $\mathbb{R}^{m}$ and let there exist a constant $\kappa>0$ and two sequences $\left(v_{n}\right)_{n \in \mathbb{N}}$ and $\left(w_{n}\right)_{n \in \mathbb{N}}$ such that $\left(v_{n}\right)_{n \in \mathbb{N}} \in L^{1}(\Omega), v \in L^{1}(\Omega)$ and

$$
v_{n} \rightarrow v \text {, weakly in } L^{1}(\Omega) \text {, as } n \rightarrow \infty,
$$

$\left(w_{n}\right) \in L^{\infty}(\Omega), w \in L^{\infty}(\Omega)$, and for all $n \in \mathbb{N},\left|w_{n}\right| \leq \kappa$ with

$$
w_{n} \rightarrow w \text {, almost everywhere in } \Omega \text {, as } n \rightarrow \infty \text {. }
$$

Then, $\lim _{n \rightarrow \infty}\left\|v_{n}\left(w_{n}-w\right)\right\|_{L^{1}(\Omega)}=0$, and

$$
v_{n} w_{n} \rightarrow v w, \text { weakly in } L^{1}(\Omega), \text { as } n \rightarrow \infty \text {. }
$$


Proof. The proof of this classical result in measure theory is based on the DunfordPettis and Egorov theorems.

All the material required for the convergence proof is now gathered. The following proposition gives, under additional assumptions on the kernels and on the mesh, some estimates which will prove useful to estimate the error in view of Theorem 2.3 .

Proposition 3.6. Let the coagulation kernel a and fragmentation kernel $b$ satisfy property (4) and let the mesh be regular in the sense of (12) or (13); the time step $\Delta t$ also satisfies (22).

Assume that the initial datum $f^{\text {in }}$ is bounded in $L_{\text {loc }}^{\infty}\left(\mathbb{R}^{+}\right)$. Then, the approximate solution $f^{h}$ and the exact solution $f$ to (11) are essentially bounded in $(0, T) \times(0, R)$,

$$
\left\|f^{h}\right\|_{L^{\infty}((0, T) \times(0, R))} \leq C(T, R), \quad\|f\|_{L^{\infty}((0, T) \times(0, R))} \leq C(T, R) .
$$

Moreover, if the kernels $a$ and $b$ satisfy (25) and the initial datum $f^{\text {in }}$ satisfies the smoothness condition (26). Then, there exists a positive constant $C(T, R)$ such that

$$
\|f(t)\|_{W^{1, \infty}(0, R)} \leq C(T, R),
$$

where $f$ is the exact solution to (11).

Proof. We prove a priori boundedness for the solution $f$ to the continuous equation (11) only. The proof in the discrete case is similar to the one for the nonnegativity of $f^{h}$. Integrating equation (11) with respect to time gives

$$
\begin{aligned}
f(t, x)= & f^{\text {in }}(x)+\int_{0}^{t}-\frac{1}{x} \frac{\partial \mathcal{C}^{R}}{\partial x}(s, x)+\frac{1}{x} \frac{\partial \mathcal{F}^{R}}{\partial x}(s, x) d s \\
\leq & f^{\text {in }}(x)+\frac{1}{2} \int_{0}^{t} \int_{0}^{x} a\left(x^{\prime}, x-x^{\prime}\right) f\left(s, x^{\prime}\right) f\left(s, x-x^{\prime}\right) d x^{\prime} d s \\
& +\int_{0}^{t} \int_{x}^{R} b\left(x^{\prime}-x, x\right) f\left(s, x^{\prime}\right) d x^{\prime} d s \\
\leq & f^{\text {in }}(x)+\|b\|_{L^{\infty}}\|f\|_{\infty, 1} t+\frac{\|a\|_{L^{\infty}}}{2}\|f\|_{\infty, 1} \int_{0}^{t} \sup _{y \in(0, R)} f(s, y) d x
\end{aligned}
$$

where $\|f\|_{\infty, 1}$ denotes the norm of $f$ in $L^{\infty}\left(0, T ; L^{1}(0, R)\right)$. Then, Gronwall's lemma enables us to conclude the proof.

We turn to the proof of estimate (43). First, we integrate equation (11) with respect to time, divide it by $x$ and next differentiate it with respect to volume variable $x$ (using formulae (9) and (10) for the coagulation and fragmentation terms). Then, taking the maximum value over all possible values of $x$, it yields

$$
\begin{aligned}
\left\|\frac{\partial f}{\partial x}(t)\right\|_{L^{\infty}}= & \left\|\frac{\partial f^{\text {in }}}{\partial x}\right\|_{L^{\infty}}+\left\{\frac{3}{2}\|a\|_{W^{1, \infty}}\|f\|_{\infty, 1}\|f\|_{L^{\infty}}+\frac{1}{2}\|a\|_{L^{\infty}}\|f\|_{L^{\infty}}^{2}\right. \\
& \left.+\frac{3}{2}\|b\|_{L^{\infty}}\|f\|_{L^{\infty}}+\|b\|_{W^{1, \infty}}\left(\|f\|_{\infty, 1}+\frac{1}{2} R\|f\|_{L^{\infty}}\right)\right\} t \\
& +\frac{3}{2}\left(\|a\|_{L^{\infty}}\|f\|_{\infty, 1}+\|b\|_{L^{\infty}}\right) \int_{0}^{t}\left\|\frac{\partial f}{\partial x}(s)\right\|_{L^{\infty}} d s
\end{aligned}
$$

and, again Gronwall's lemma allows us to conclude. 
Note that, in this proof, $f \in L^{\infty}\left(0, T ; L^{1}(0, R)\right)$ has been extensively used. Indeed, Theorem 2.2 gives this information under the same (or weaker) assumptions as those made in Proposition 3.6. We underline that, of course, Theorem 2.2 is proven independently of this proposition.

\section{Convergence of the numerical SOlution}

Proving Theorem 2.2 is achieved by interpreting the sequence $f_{i}^{n}$ built from the numerical scheme as a sequence of step functions $f^{h}$ depending on the mesh size $h$ and on $\Delta t$. Properties (in particular weak compactness) of this sequence have been stated in detail in the previous section. The proof is now reduced to writing the discrete coagulation and fragmentation operators in terms of $f^{h}$ and proving (weak) convergence for these expressions towards the continuous coagulation and fragmentation operators (5)-(6) . This is provided by Lemma 4.1 below. Then the usual finite volumes techniques allow us to conclude.

The following notations will be used throughout the analysis of the numerical scheme. First, several point approximations are defined:

$$
\begin{aligned}
& X^{h}: x \in(0, R) \quad \rightarrow \quad X^{h}(x)=\sum_{i=0}^{I^{h}} x_{i} \chi_{\Lambda_{i}^{h}}(x), \quad \text { (midpoint approximation) } \\
& \Xi^{h}: x \in(0, R) \quad \rightarrow \quad \Xi^{h}(x)=\sum_{i=0}^{I^{h}} x_{i+1 / 2} \chi_{\Lambda_{i}^{h}}(x), \quad \text { (right endpoint approximation) } \\
& \xi^{h}: x \in(0, R) \quad \rightarrow \quad \xi^{h}(x)=\sum_{i=0}^{I^{h}} x_{i-1 / 2} \chi_{\Lambda_{i}^{h}}(x), \quad \text { (left endpoint approximation) }
\end{aligned}
$$

and

$$
\Theta^{h}:(x, u) \in(0, R)^{2} \rightarrow \Theta^{h}(x, u)=\sum_{i=0}^{I^{h}} \sum_{j=0}^{i} x_{\gamma_{i, j}} \chi_{\Lambda_{i}^{h}}(x) \chi_{\Lambda_{j}^{h}}(u)
$$

At this stage, note that $\left(X^{h}\right)_{h},\left(\Xi^{h}\right)_{h}$ and $\left(\Theta^{h}\right)_{h}$ converge pointwisely: for all $x \in(0, R)$,

$$
X^{h}(x) \rightarrow x, \quad \Xi^{h}(x) \rightarrow x,
$$

as $h \rightarrow 0$ and for all $(x, u) \in(0, R)^{2}$, we have

$$
\left\{\begin{array}{lll}
\Theta^{h}(x, u) & \rightarrow x-u & \text { if } x \geq u \\
\Theta^{h}(x, u) & \rightarrow 0 & \text { if } x \leq u
\end{array}\right.
$$

Then, the proof of Theorem 2.2 is based on the following lemma.

Lemma 4.1. Let us define the approximations of the coagulation and fragmentation terms according to:

$$
\begin{aligned}
\mathcal{C}^{h}(t, x) & =\int_{0}^{R} \int_{0}^{R} \chi_{\left[0, \Xi^{h}(x)\right]}(u) \chi_{\left[\Theta^{h}(x, u), R\right]}(v) X^{h}(u) a^{h}(u, v) f^{h}(t, u) f^{h}(t, v) d v d u, \\
\mathcal{F}^{h}(t, x) & =\int_{0}^{R} \int_{0}^{R} \chi_{\left[0, \Xi^{h}(x)\right]}(u) \chi_{\left[\Xi^{h}(x)-u, R-u\right]}(v) X^{h}(u) b^{h}(u, v) f^{h}(t, u+v) d v d u .
\end{aligned}
$$


There exists a subsequence of $\left(f^{h}\right)_{h}$, such that

$$
\begin{aligned}
& \mathcal{C}^{h} \rightarrow \mathcal{C}_{n c}^{R} \text { in } L^{1}((0, T) \times(0, R)), \quad \text { as } h \rightarrow 0, \\
& \mathcal{F}^{h} \rightarrow \mathcal{F}_{n c}^{R} \text { in } \quad L^{1}((0, T) \times(0, R)), \quad \text { as } h \rightarrow 0 .
\end{aligned}
$$

In addition, $\mathcal{C}^{h}(\cdot, R)$ converges weakly to $\mathcal{C}_{n c}^{R}(\cdot, R)$ in $L^{1}(0, T)$.

Proof. We consider the sequences $\left(f^{h}\right)_{h},\left(a^{h}\right)_{h}$ and $\left(b^{h}\right)_{h}$ extracted according to the procedure sketched in Remark 3.4. Then, obviously for all $(t, x) \in(0, T) \times(0, R)$ and almost all $(u, v) \in(0, R) \times(0, R)$, the sequence $X^{h}(.) a^{h}(., v)$ is bounded in $L^{\infty}(0, R)$ and

$$
\chi_{\left[0, \Xi^{h}(x)\right]}(u) \chi_{\left[\Theta^{h}(x, u), R\right]}(v) X^{h}(u) a^{h}(u, v) \rightarrow \chi_{[0, x]}(u) \chi_{[x-u, R]}(v) u a(u, v),
$$

as $h$ goes to 0 . Thus, applying Lemma 3.5, it yields

$$
\begin{gathered}
\int_{0}^{R} \chi_{\left[0, \Xi^{h}(x)\right]}(u) \chi_{\left[\Theta^{h}(x, u), R\right]}(v) X^{h}(u) a^{h}(u, v) f^{h}(t, u) d u \\
\rightarrow \int_{0}^{x} \chi_{[x-u, R]}(v) u a(u, v) f(t, u) d u
\end{gathered}
$$

for each $t, x$, and almost every $v$. The same argument is used to prove the pointwise convergence of $\mathcal{C}^{h}$. Indeed, for each $x$ and $t$ and for almost every $v$ (44) holds true, and since $f^{h}$ converges weakly, Lemma 3.5 applies again and gives

$$
\mathcal{C}^{h}(t, x) \rightarrow \mathcal{C}_{\mathrm{nc}}^{R}(t, x)
$$

for every $(t, x) \in[0, T] \times[0, R]$. This pointwise convergence obviously implies weak convergence for $\mathcal{C}^{h}$ and for the boundary value $\mathcal{C}^{h}(\cdot, R)$.

The convergence study of $\mathcal{F}^{h}$ is similar to that of $\mathcal{C}^{h}$, observing that

$$
\mathcal{F}^{h}(t, x)=\int_{0}^{R} \int_{0}^{R} \chi_{\left[0, \Xi^{h}(x)\right]}(u) \chi_{\left[\Xi^{h}(x), R\right]}(v) X^{h}(u) b^{h}(u, v-u) f^{h}(t, v) d v d u
$$

and recalling that $b^{h}(u, v-u)$ converges to $b(u, v-u)$ for almost every $0 \leq u \leq$ $v \leq R$.

To make the importance of this lemma clear, it may be useful to mention that $\mathcal{C}^{h}(t, x)\left(\right.$ resp. $\left.\mathcal{F}^{h}(t, x)\right)$ actually coincide with $\mathcal{C}_{i}^{n}\left(\right.$ resp. $\left.\mathcal{F}_{i}^{n}\right)$ whenever $t \in \tau_{n}$ and $x \in \Lambda_{i}^{h}$. This will be proven in the sequel.

Now, we turn to the proof of Theorem 2.2. We consider a test function $\varphi \in$ $C^{1}([0, T) \times[0, R])$, which is compactly supported. On the one hand, we observe that, for $\Delta t$ small enough, the support of $\varphi$ with respect to the time variable satisfies $\operatorname{Supp}_{t} \varphi \subset\left[0, t_{N-1}\right]$. On the other hand, we define the finite volume (in time) and left endpoint (in space) approximation of $\varphi$ on $\tau_{n} \times \Lambda_{i}^{h}$ by

$$
\varphi_{i}^{n}:=\frac{1}{\Delta t} \int_{t_{n}}^{t_{n+1}} \varphi\left(t, x_{i-1 / 2}\right) d t .
$$


Then, multiplying equation (16) by $\varphi_{i}^{n}$ and summing over $n \in\{0, \ldots, N-1\}$ and $i \in\left\{0, \ldots, I^{h}\right\}$, yields by a discrete integration by parts,

$$
\begin{aligned}
& \sum_{n=0}^{N-1} \sum_{i=0}^{I^{h}} \Delta x_{i} x_{i} f_{i}^{n+1}\left(\varphi_{i}^{n+1}-\varphi_{i}^{n}\right) \\
& +\sum_{n=0}^{N-1} \sum_{i=0}^{I^{h}-1} \Delta t\left[\mathcal{C}_{i+1 / 2}^{n}-\mathcal{F}_{i+1 / 2}^{n}\right]\left(\varphi_{i+1}^{n}-\varphi_{i}^{n}\right) \\
& +\sum_{i=0}^{I^{h}} \Delta x_{i} x_{i} f_{i}^{\text {in }} \varphi_{i}^{0}-\sum_{n=0}^{N-1} \Delta t \mathcal{C}_{I^{h}+1 / 2}^{n} \varphi_{I^{h}}^{n}=0,
\end{aligned}
$$

where the boundary and initial value properties (19)-(20) have been used. The first and third terms in the left hand side of equation (45) can be written in terms of function $f^{h}$ :

$$
\begin{aligned}
& \sum_{n=0}^{N-1} \sum_{i=0}^{I^{h}} \Delta x_{i} x_{i} f_{i}^{n+1}\left(\varphi_{i}^{n+1}-\varphi_{i}^{n}\right)+\sum_{i=0}^{I^{h}} \Delta x_{i} x_{i} f_{i}^{\text {in }} \varphi_{i}^{0} \\
& =\sum_{n=0}^{N-1} \sum_{i=0}^{I^{h}} \int_{\tau_{n+1}} \int_{\Lambda_{i}^{h}} X^{h}(x) f^{h}(t, x) \frac{\varphi\left(t, \xi^{h}(x)\right)-\varphi\left(t-\Delta t, \xi^{h}(x)\right)}{\Delta t} d x d t \\
& \quad+\sum_{i=0}^{I^{h}} \int_{\Lambda_{i}^{h}} X^{h}(x) f^{h}(0, x) \frac{1}{\Delta t} \int_{0}^{\Delta t} \varphi\left(t, \xi^{h}(x)\right) d t d x \\
& =\int_{\Delta t}^{T} \int_{0}^{R} X^{h}(x) f^{h}(t, x) \frac{\varphi\left(t, \xi^{h}(x)\right)-\varphi\left(t-\Delta t, \xi^{h}(x)\right)}{\Delta t} d x d t \\
& \quad+\int_{0}^{R} X^{h}(x) f^{h}(0, x) \frac{1}{\Delta t} \int_{0}^{\Delta t} \varphi\left(t, \xi^{h}(x)\right) d t d x .
\end{aligned}
$$

We first treat the last term of the former equality. On the one hand, $X^{h}(x)$ converges pointwise in $[0, R]$ whereas $f^{h}(0, \cdot)$ is a finite volume approximation of $f^{\text {in }}$ and, henceforth, converges strongly in $L^{1}(0, R)$. On the other hand, since $\varphi$ is continuously differentiable with compact support, its derivatives are bounded and, therefore, the following convergence is uniform with respect to $t, x$ as $\max \{h, \Delta t\}$ goes to 0 :

Consequently, it first yields

$$
\frac{1}{\Delta t} \int_{0}^{\Delta t} \varphi\left(t, \xi^{h}(x)\right) d t \rightarrow \varphi(0, x) .
$$

$$
\int_{0}^{R} X^{h}(x) f^{h}(0, x) \frac{1}{\Delta t} \int_{0}^{\Delta t} \varphi\left(t, \xi^{h}(x)\right) d t d x \rightarrow \int_{0}^{R} x f^{\text {in }}(x) \varphi(0, x) d x,
$$

as $\max \{h, \Delta t\}$ goes to 0 .

To deal with the first term in (46), a Taylor expansion of the smooth function $\varphi$ is performed, which finally yields

$$
\frac{\varphi\left(t, \xi^{h}(x)\right)-\varphi\left(t-\Delta t, \xi^{h}(x)\right)}{\Delta t} \rightarrow \frac{\partial \varphi}{\partial t}(t, x)
$$

uniformly as $\max \{h, \Delta t\}$ goes to 0 , while $\chi_{[\Delta t, T]}(t) X^{h}(x)$ converges pointwise to $\chi_{[0, T]}(t) x$. Applying Lemma 3.5, together with Proposition [3.3. entails that the 
first term on the right hand side of (46) converges to

$$
\int_{0}^{T} \int_{0}^{R} x f(t, x) \frac{\partial \varphi}{\partial t}(t, x) d x d t
$$

as $\max \{h, \Delta t\}$ goes to 0 .

Consider now the coagulation and fragmentation terms. As mentioned above, the approximations $\mathcal{C}^{h}$ and $\mathcal{F}^{h}$ coincide with the discrete coagulation and fragmentation terms. Indeed, on the one hand, for $t \in \tau_{n}$ and $x \in \Lambda_{i}^{h}$,

$$
\begin{aligned}
\mathcal{C}^{h}(t, x) & =\int_{0}^{x_{i+1 / 2}} \int_{\Theta^{h}(x, u)}^{R} X^{h}(u) a^{h}(u, v) f^{h}(t, u) f^{h}(t, v) d v d u \\
& =\sum_{j=0}^{i} \int_{\Lambda_{j}^{h}} d u \sum_{k=\gamma_{i, j}}^{I^{h}} \int_{\Lambda_{k}^{h}} d v x_{j} a_{j, k} f_{j}^{n} f_{k}^{n} \\
& =\mathcal{C}_{i+1 / 2}^{n},
\end{aligned}
$$

where we have used that, for $x \in \Lambda_{i}^{h}$ and $u \in \Lambda_{j}^{h}, \Theta^{h}(x, u)=x_{\gamma_{i, j}}$. On the other hand, for the fragmentation operator

$$
\begin{aligned}
\mathcal{F}^{h}(t, x) & =\int_{0}^{x_{i+1 / 2}} \int_{x_{i+1 / 2}}^{R} X^{h}(u) b^{h}(u, v-u) f^{h}(t, v) d v d u \\
& =\sum_{j=0}^{i} \int_{\Lambda_{j}^{h}} d u \sum_{k=i+1}^{I^{h}} \int_{\Lambda_{k}^{h}} d v x_{j} b_{j, k} f_{k}^{n} \\
& =\mathcal{F}_{i+1 / 2}^{n} .
\end{aligned}
$$

Consequently, it is straightforward to write the second and fourth terms of the left hand side of equation (45) in terms of $\mathcal{C}^{h}$ and $\mathcal{F}^{h}$ :

$$
\begin{aligned}
& \sum_{n=0}^{N-1} \sum_{i=0}^{I^{h}-1} \Delta t\left[\mathcal{C}_{i+1 / 2}^{n}-\mathcal{F}_{i+1 / 2}^{n}\right]\left(\varphi_{i+1}^{n}-\varphi_{i}^{n}\right)-\sum_{n=0}^{N-1} \Delta t \mathcal{C}_{I^{h}+1 / 2}^{n} \varphi_{I^{h}}^{n} \\
= & \sum_{n=0}^{N-1} \sum_{i=0}^{I^{h}-1} \int_{\tau_{n}} \int_{\Lambda_{i}^{h}}\left[\mathcal{C}_{i+1 / 2}^{n}-\mathcal{F}_{i+1 / 2}^{n}\right] \frac{1}{\Delta x_{i}}\left(\varphi\left(t, x_{i+1 / 2}\right)-\varphi\left(t, x_{i-1 / 2}\right)\right) d x d t \\
- & \sum_{n=0}^{N-1} \int_{\tau_{n}} \mathcal{C}_{I^{h}+1 / 2}^{n} \varphi\left(t, R-\Delta x_{I^{h}}\right) d t \\
= & \int_{0}^{T} \int_{0}^{R-\Delta x_{I^{h}}}\left[\mathcal{C}^{h}(t, x)-\mathcal{F}^{h}(t, x)\right] \frac{\partial \varphi}{\partial x}(t, x) d x d t \\
- & \int_{0}^{T} \mathcal{C}^{h}(t, R) \varphi\left(t, R-\Delta x_{I^{h}}\right) d t .
\end{aligned}
$$

Therefore, the weak compactness result given by Lemma 4.1 implies the convergence of the right hand side of the latter equality to the corresponding terms in (24), as expected.

\section{ERROR ESTIMATES ON THE NUMERICAL SOLUTION}

The error estimate is performed by giving a priori estimates on the difference $f^{h}-f$, where $f^{h}$ is built thanks to the numerical scheme and $f$ is the exact solution 
to (11). The difference $f^{h}-f$ is obviously a solution to the difference of equations (16) and (11) respectively divided by $x_{i}$ and $x$. A mere integration by parts enables us to give to equation (11) divided by $x$ a convenient form. Lemma 5.1 shows that summations by parts can yield a similar result in the discrete case. Then, estimating the difference $f^{h}-f$ is easy thanks to Proposition 3.6.

When the mesh is uniform, that is, $\Delta x_{i}=h$ for all $i \in\left\{0, \ldots, I^{h}\right\}$, the discrete coagulatation and fragmentation terms read

$$
\begin{aligned}
-\frac{\mathcal{C}_{i+\frac{1}{2}}^{n}-\mathcal{C}_{i-\frac{1}{2}}^{n}}{h} & =h \sum_{j=0}^{i-1} x_{j} a_{j, i-j-1} f_{j}^{n} f_{i-j-1}^{n}-h \sum_{j=0}^{I^{h}} x_{i} a_{i, j} f_{i}^{n} f_{j}^{n}, \\
\frac{\mathcal{F}_{i+\frac{1}{2}}^{n}-\mathcal{F}_{i-\frac{1}{2}}^{n}}{h} & =-h \sum_{j=0}^{i-1} x_{j} b_{j, i} f_{i}^{n}+h \sum_{j=i+1}^{I^{h}} x_{i} b_{i, j} f_{j}^{n} .
\end{aligned}
$$

The following lemma gives a simplified expression of these variation rates.

Lemma 5.1. Assume that $a$ and $b$ satisfy (41) and (25) respectively and consider a uniform mesh, that is, $\Delta x_{i}=h$ for all $i$. We also assume that the initial datum $f^{\text {in }}$ is bounded in $L^{\infty}\left(\mathbb{R}^{+}\right)$. Let $(s, x) \in \tau_{n}^{h} \times \Lambda_{i}^{h}$, with $n \in\{0, \ldots, N-1\}, i \in\left\{0, \ldots, I^{h}\right\}$. Then

$$
\begin{aligned}
-\frac{\mathcal{C}_{i+\frac{1}{2}}^{n}-\mathcal{C}_{i-\frac{1}{2}}^{n}}{x_{i} h}= & \frac{1}{2} \int_{0}^{\xi^{h}(x)} a^{h}\left(x^{\prime}, x-\Xi^{h}\left(x^{\prime}\right)\right) f^{h}\left(s, x^{\prime}\right) f^{h}\left(s, x-\Xi^{h}\left(x^{\prime}\right)\right) d x^{\prime} \\
& -\int_{0}^{R} a^{h}\left(x, x^{\prime}\right) f^{h}\left(s, x^{\prime}\right) d x^{\prime} f^{h}(s, x)+\varepsilon_{\mathrm{c}}^{h}(x) \\
\frac{\mathcal{F}_{i+\frac{1}{2}}^{n}-\mathcal{F}_{i-\frac{1}{2}}^{n}}{x_{i} h}= & -\frac{1}{2} \int_{h}^{\xi^{h}(x)} b^{h}\left(x^{\prime}, x-x^{\prime}\right) d x^{\prime} f^{h}(s, x) \\
& +\int_{\Xi^{h}(x)}^{R} b^{h}\left(x, x^{\prime}-x\right) f^{h}\left(s, x^{\prime}\right) d x^{\prime}+\varepsilon_{\mathrm{f}}^{h}(x)
\end{aligned}
$$

where $\varepsilon_{\mathrm{c}}^{h}$ and $\varepsilon_{\mathrm{f}}^{h}$ denote first order terms with respect to $h$ in the strong $L^{1}$ topology:

$$
\begin{aligned}
\left\|\varepsilon_{\mathrm{c}}^{h}\right\|_{L^{1}} & \leq \frac{R}{4}\left\|f^{h}\right\|_{L^{\infty}}^{2}\|a\|_{L^{\infty}} h, \\
\left\|\varepsilon_{\mathrm{f}}^{h}\right\|_{L^{1}} & \leq\left(\frac{R}{2}\left\|f^{h}\right\|_{L^{\infty}}+\left\|f^{h}\right\|_{\infty, 1}\right)\|b\|_{L^{\infty}} h .
\end{aligned}
$$

Proof. First, the variation rates have to be written in such a way that the volume $x_{i}$ is factored out. To this aim, we consider for instance the fragmentation term: since the mesh is uniform, it holds that $x_{i+\frac{1}{2}}-x_{j}=x_{i-j}$ and we have, for $i \geq 1$,

$$
\begin{aligned}
-h \sum_{j=1}^{i-1} x_{j} b_{j, i} f_{i}^{n} & =h \sum_{j=1}^{i-1} x_{i-j} b_{j, i} f_{i}^{n}-x_{i+\frac{1}{2}} h \sum_{j=1}^{i-1} b_{j, i} f_{i}^{n} \\
& =h \sum_{j=1}^{i-1} x_{j} b_{i-j, i} f_{i}^{n}-x_{i+\frac{1}{2}} h \sum_{j=1}^{i-1} b_{j, i} f_{i}^{n} .
\end{aligned}
$$

Thus, using the symmetry property (35) of $b_{i, j}$,

$$
-h \sum_{j=1}^{i-1} x_{j} b_{j, i} f_{i}^{n}=h \sum_{j=1}^{i-1} x_{j} b_{j, i} f_{i}^{n}-x_{i+\frac{1}{2}} h \sum_{j=1}^{i-1} b_{j, i} f_{i}^{n},
$$


which finally gives

$$
h \sum_{j=1}^{i-1} x_{j} b_{j, i} f_{i}^{n}=\frac{h}{2} x_{i+\frac{1}{2}} \sum_{j=1}^{i-1} b_{j, i} f_{i}^{n}
$$

and for (48)

$$
\begin{aligned}
& \frac{\mathcal{F}_{i+\frac{1}{2}}^{n}-\mathcal{F}_{i-\frac{1}{2}}^{n}}{h x_{i}} \\
& =h\left[-\frac{x_{0}}{x_{i}} b_{0, i} f_{i}^{n}-\frac{x_{i+\frac{1}{2}}}{x_{i}} \frac{1}{2} \sum_{j=1}^{i-1} b_{j, i} f_{i}^{n}+\sum_{j=i+1}^{I^{h}} b_{i, j} f_{j}^{n}\right] \\
& =h\left[-\frac{x_{0}}{x_{i}} b_{0, i} f_{i}^{n}-\frac{h}{4 x_{i}} \sum_{j=1}^{i-1} b_{j, i} f_{i}^{n}-\frac{1}{2} \sum_{j=1}^{i-1} b_{j, i} f_{i}^{n}+\sum_{j=i+1}^{I^{h}} b_{i, j} f_{j}^{n}\right]
\end{aligned}
$$

since $x_{i+\frac{1}{2}}=x_{i}+\frac{h}{2}$. We have used convention (34), so that $b_{0, i}$ is equal to zero whenever $i=0$. We define

$$
\varepsilon_{\mathrm{f}}^{h}(x)=-h \frac{x_{0}}{x_{i}} b_{0, i} f_{i}^{n}-\frac{h^{2}}{4 x_{i}} \sum_{j=1}^{i-1} b_{j, i} f_{i}^{n}
$$

and then get (50). Moreover, estimating the $x$ integral of this term is equivalent to estimating the following sum:

$$
h \sum_{i=0}^{I^{h}}\left|h \frac{x_{0}}{x_{i}} b_{0, i} f_{i}^{n}+\frac{h^{2}}{4 x_{i}} \sum_{j=1}^{i-1} b_{j, i} f_{i}^{n}\right| .
$$

The first term in this sum is bounded if one remarks that $x_{0} / x_{i} \leq 1$ while the second one is bounded by noting that

$$
\frac{h^{2}}{4 x_{i}} \sum_{j=1}^{i-1} b_{j, i} \leq h\|b\|_{L^{\infty}} \frac{(i-1) h}{4 x_{i}} \leq \frac{h}{4}\|b\|_{L^{\infty}} .
$$

Hence, applying Proposition 3.6. the approximate solution is bounded in $L^{\infty}$ and estimate (52) easily follows.

Now, turning to equation (47) and remarking that $x_{i-\frac{1}{2}}-x_{j}=x_{i-j-1}$, we similarly prove that

$$
-\frac{\mathcal{C}_{i+\frac{1}{2}}^{n}-\mathcal{C}_{i-\frac{1}{2}}^{n}}{x_{i} h}=\frac{x_{i-\frac{1}{2}}}{2 x_{i}} h \sum_{j=0}^{i-1} a_{j, i-j-1} f_{j}^{n} f_{i-j-1}^{n}-h \sum_{j=0}^{I^{h}} a_{i, j} f_{i}^{n} f_{j}^{n},
$$

which gives expression (49) by setting

$$
\varepsilon_{\mathrm{c}}^{h}(x)=-\frac{h^{2}}{4 x_{i}} \sum_{j=0}^{i-1} a_{j, i-j-1} f_{j}^{n} f_{i-j-1}^{n}
$$

and the estimate (51) is obtained in the same way as (52). 
Therefore, both terms (51) and (52) have to be compared to the explicit formulation of the continuous coagulation and fragmentation terms

$$
\begin{aligned}
-\frac{1}{x} \frac{\partial \mathcal{C}^{R}}{\partial x}(t, x)= & \frac{1}{2} \int_{0}^{x} a\left(x^{\prime}, x-x^{\prime}\right) f\left(t, x^{\prime}\right) f\left(t, x-x^{\prime}\right) d x^{\prime} \\
& -\int_{0}^{R} a\left(x, x^{\prime}\right) f\left(t, x^{\prime}\right) d x^{\prime} f(t, x), \\
\frac{1}{x} \frac{\partial \mathcal{F}^{R}}{\partial x}(t, x)= & -\frac{1}{2} \int_{0}^{x} b\left(x^{\prime}, x-x^{\prime}\right) d x^{\prime} f(t, x) \\
& +\int_{x}^{R} b\left(x, x^{\prime}-x\right) f\left(t, x^{\prime}\right) d x^{\prime} .
\end{aligned}
$$

Using formulae (49), (50), (53) and (54), equation (111) and the scheme (16), we easily get for $t \in \tau_{n}$,

$$
\begin{aligned}
\int_{0}^{R} \mid f^{h}( & t, x)-f(t, x)\left|d x \leq \int_{0}^{R}\right| f^{h}(0, x)-f^{\mathrm{in}}(x) \mid d x \\
& +\sum_{\alpha=1}^{4}\left[\mathcal{E}_{\mathrm{c}, \alpha}^{h}+\mathcal{E}_{\mathrm{f}, \alpha}^{h}\right]+\mathcal{E}_{\mathrm{t}, n}^{h}+\left\|\varepsilon_{\mathrm{c}}^{h}\right\|_{L^{1}}+\left\|\varepsilon_{\mathrm{f}}^{h}\right\|_{L^{1}},
\end{aligned}
$$

where $\left(\mathcal{E}_{c, \alpha}^{h}\right)_{\alpha=1, . ., 4}$ are error terms related to the coagulation operator

$$
\begin{aligned}
\mathcal{E}_{\mathrm{c}, 1}^{h}= & \frac{1}{2} \int_{0}^{t} \int_{0}^{R} \int_{0}^{\xi^{h}(x)} \mid a^{h}\left(x^{\prime}, x-\Xi^{h}\left(x^{\prime}\right)\right) f^{h}\left(s, x^{\prime}\right) f^{h}\left(s, x-\Xi^{h}\left(x^{\prime}\right)\right) \\
& -a\left(x^{\prime}, x-\Xi^{h}\left(x^{\prime}\right)\right) f\left(s, x^{\prime}\right) f\left(s, x-\Xi^{h}\left(x^{\prime}\right)\right) \mid d x^{\prime} d x d s \\
\mathcal{E}_{\mathrm{c}, 2}^{h}= & \frac{1}{2} \int_{0}^{t} \int_{0}^{R} \int_{0}^{\xi^{h}(x)} \mid a\left(x^{\prime}, x-\Xi^{h}\left(x^{\prime}\right)\right) f\left(s, x^{\prime}\right) f\left(s, x-\Xi^{h}\left(x^{\prime}\right)\right) \\
& -a\left(x^{\prime}, x-x^{\prime}\right) f\left(s, x^{\prime}\right) f\left(s, x-x^{\prime}\right) \mid d x^{\prime} d x d s \\
\mathcal{E}_{\mathrm{c}, 3}^{h}= & \frac{1}{2} \int_{0}^{t} \int_{0}^{R} \int_{\xi^{h}(x)}^{x} a\left(x^{\prime}, x-x^{\prime}\right) f\left(s, x^{\prime}\right) f\left(s, x-x^{\prime}\right) d x^{\prime} d x d s
\end{aligned}
$$

and

$\mathcal{E}_{\mathrm{c}, 4}^{h}=\int_{0}^{t} \int_{0}^{R} \int_{0}^{R}\left|a^{h}\left(x, x^{\prime}\right) f^{h}(s, x) f^{h}\left(s, x^{\prime}\right)-a\left(x, x^{\prime}\right) f(s, x) f\left(s, x^{\prime}\right)\right| d x^{\prime} d x d s$, whereas $\left(\mathcal{E}_{\mathbf{f}, \alpha}^{h}\right)_{\alpha=1, . ., 4}$ are error terms related to the fragmentation operator $\mathcal{E}_{\mathrm{f}, 1}^{h}=\frac{1}{2} \int_{0}^{t} \int_{0}^{R} \int_{h}^{\xi^{h}(x)}\left|b^{h}\left(x^{\prime}, x-x^{\prime}\right) f^{h}(s, x)-b\left(x^{\prime}, x-x^{\prime}\right) f(s, x)\right| d x^{\prime} d x d s$

$\mathcal{E}_{\mathrm{f}, 2}^{h}=\frac{1}{2} \int_{0}^{t} \int_{0}^{R} \int_{[0, h] \cup\left[\xi^{h}(x), x\right]} b^{h}\left(x^{\prime}, x-x^{\prime}\right) f^{h}(s, x) d x^{\prime} d x d s$ $\mathcal{E}_{\mathrm{f}, 3}^{h}=\int_{0}^{t} \int_{0}^{R} \int_{\Xi^{h}(x)}^{R}\left|b^{h}\left(x, x^{\prime}-x\right) f^{h}\left(s, x^{\prime}\right)-b\left(x, x^{\prime}-x\right) f\left(s, x^{\prime}\right)\right| d x^{\prime} d x d s$

and

$$
\mathcal{E}_{\mathrm{f}, 4}^{h}=\int_{0}^{t} \int_{0}^{R} \int_{x}^{\Xi^{h}(x)} b^{h}\left(x, x^{\prime}-x\right) f^{h}\left(s, x^{\prime}\right) d x^{\prime} d x d s .
$$


Finally, $\mathcal{E}_{\mathrm{t}, n}^{h}$ is the error due to the time discretization:

$$
\begin{aligned}
\mathcal{E}_{\mathrm{t}, n}^{h}= & \int_{t_{n}}^{t}\left\{\int _ { 0 } ^ { R } \left[\frac{1}{2} \int_{0}^{\xi^{h}(x)} a^{h}\left(x^{\prime}, x-\Xi^{h}\left(x^{\prime}\right)\right) f^{h}\left(s, x^{\prime}\right) f^{h}\left(s, x-\Xi^{h}\left(x^{\prime}\right)\right) d x^{\prime}\right.\right. \\
& +\int_{0}^{R} a^{h}\left(x, x^{\prime}\right) f^{h}(s, x) f^{h}\left(s, x^{\prime}\right) d x^{\prime} \\
& +\varepsilon_{\mathrm{c}}^{h}(x)+\frac{1}{2} \int_{h}^{\xi^{h}(x)} b^{h}\left(x^{\prime}, x-x^{\prime}\right) f^{h}(s, x) d x^{\prime} \\
& \left.\left.+\int_{\Xi^{h}(x)}^{R} b^{h}\left(x, x^{\prime}-x\right) f^{h}\left(s, x^{\prime}\right) d x^{\prime}+\varepsilon_{\mathrm{f}}^{h}(x)\right] d x\right\} d s .
\end{aligned}
$$

On the one hand, the error given by $\mathcal{E}_{\mathrm{c}, 2}^{h}$ is estimated from the smoothness of the kernel $a$ and of the solution $f$ to (11). Indeed, since in this section $a$ is taken in $W_{l o c}^{1, \infty}$, we have for all $x \in(0, R)$ and $x^{\prime} \in \Lambda_{i}^{h}$

$$
\left|a\left(x^{\prime}, x-x_{i}\right)-a\left(x^{\prime}, x-x^{\prime}\right)\right| \leq\|a\|_{W^{1, \infty}} h
$$

and applying Proposition 3.6, the $W^{1, \infty}$ estimate (43) on $f$ gives

$$
\left|f\left(t, x-x_{i}\right)-f\left(t, x-x^{\prime}\right)\right| \leq\|f(t, .)\|_{W^{1, \infty}} h .
$$

Thus, it yields

$$
\mathcal{E}_{\mathrm{c}, 2}^{h} \leq \frac{R^{2}}{4}\|f\|_{\infty}\left(\|a\|_{W^{1, \infty}}\|f\|_{\infty}+\|a\|_{L^{\infty}}\|f\|_{L^{\infty}\left(W^{1, \infty}\right)}\right) t h .
$$

On the other hand, we consider the terms $\mathcal{E}_{\mathrm{c}, 3}^{h}, \mathcal{E}_{\mathrm{f}, 2}^{h}$ and $\mathcal{E}_{\mathrm{f}, 4}^{h}$ which are all integrals on a domain of size $t R h$. Then $L^{\infty}$ bounds on $f$ and $f^{h}$ give

$$
\mathcal{E}_{\mathrm{c}, 3}^{h} \leq\|a\|_{L^{\infty}}\|f\|_{L^{\infty}}^{2} t R h
$$

and

$$
\mathcal{E}_{f, 2}^{h}+\mathcal{E}_{f, 4}^{h} \leq 2\|b\|_{L^{\infty}}\left\|f^{h}\right\|_{L^{\infty}} t R h .
$$

The error due to the time discretization is treated similarly and it is easily seen that

$$
\mathcal{E}_{\mathrm{t}, n}^{h} \leq 2\left\{R^{2}\left\|f^{h}\right\|_{L^{\infty}}^{2}\|a\|_{L^{\infty}}+R^{2}\left\|f^{h}\right\|_{L^{\infty}}\|b\|_{L^{\infty}}+\left\|\varepsilon_{\mathrm{c}}^{h}\right\|_{L^{1}}+\left\|\varepsilon_{\mathrm{f}}^{h}\right\|_{L^{1}}\right\} \Delta t
$$

since $\left|t-t_{n}\right| \leq \Delta t$.

Finally, we turn to the estimation of terms $\mathcal{E}_{\mathrm{c}, 1}^{h}, \mathcal{E}_{\mathrm{c}, 4}^{h}, \mathcal{E}_{\mathrm{f}, 1}^{h}$ and $\mathcal{E}_{\mathrm{f}, 3}^{h}$. A detailed calculation is given for $\mathcal{E}_{c, 1}^{h}$. Estimations for the other terms are obtained by using similar arguments and details are left to the reader to check. First we perform a change of variable $x \rightarrow y=x-\Xi^{h}\left(x^{\prime}\right)$ and split $\mathcal{E}_{\mathrm{c}, 1}^{h}$ into three parts:

$$
\begin{aligned}
\mathcal{E}_{\mathrm{c}, 1}^{h} & \leq \frac{1}{2} \int_{0}^{t} \int_{0}^{R} \int_{0}^{R}\left|a^{h}\left(x^{\prime}, y\right)-a\left(x^{\prime}, y\right)\right| f\left(s, x^{\prime}\right) f(s, y) d x^{\prime} d y d s \\
& +\frac{1}{2} \int_{0}^{t} \int_{0}^{R} \int_{0}^{R} a^{h}\left(x^{\prime}, y\right)\left|f^{h}\left(s, x^{\prime}\right)-f\left(s, x^{\prime}\right)\right| f(s, y) d x^{\prime} d y d s \\
& +\frac{1}{2} \int_{0}^{t} \int_{0}^{R} \int_{0}^{R} a^{h}\left(x^{\prime}, y\right) f^{h}\left(s, x^{\prime}\right)\left|f^{h}(s, y)-f(s, y)\right| d x^{\prime} d y d s .
\end{aligned}
$$


Thus, it yields,

$$
\begin{aligned}
\mathcal{E}_{c, 1}^{h} & \leq\left(t R^{2}\|f\|_{L^{\infty}}^{2}\|a\|_{W^{1, \infty}}\right) \frac{h}{2} \\
& +\frac{R}{2}\|a\|_{L^{\infty}}\left(\left\|f^{h}\right\|_{L^{\infty}}+\|f\|_{L^{\infty}}\right) \int_{0}^{t}\left\|f^{h}(s)-f(s)\right\|_{L^{1}} d s .
\end{aligned}
$$

Similarly,

$$
\begin{aligned}
\mathcal{E}_{c, 4}^{h} & \leq\left(t R^{2}\|f\|_{L^{\infty}}^{2}\|a\|_{W^{1, \infty}}\right) \frac{h}{2} \\
& +\frac{R}{2}\|a\|_{L^{\infty}}\left(\left\|f^{h}\right\|_{L^{\infty}}+\|f\|_{L^{\infty}}\right) \int_{0}^{t}\left\|f^{h}(s)-f(s)\right\|_{L^{1}} d s .
\end{aligned}
$$

and

$$
\begin{aligned}
\mathcal{E}_{f, 1}^{h}+\mathcal{E}_{f, 3}^{h} & \leq 2\left(t R^{2}\|f\|_{L^{\infty}}\|b\|_{W^{1, \infty}}\right) h \\
& +2 R\|b\|_{L^{\infty}} \int_{0}^{t}\left\|f^{h}(s)-f(s)\right\|_{L^{1}} d s .
\end{aligned}
$$

Finally, using (55) and gathering estimates (56)-(62), we conclude thanks to Gronwall's lemma that

$$
\left\|f^{h}-f\right\|_{L^{\infty}\left(0, T ; L^{1}(0, R)\right)} \leq C(T, R)\left\{\left\|f^{h}(0, \cdot)-f^{\text {in }}\right\|_{L^{1}(0, R)}+(h+\Delta t)\right\} .
$$

To get estimate (27), one only has to remember that $f^{\text {in }}$ is taken in $W_{l o c}^{1, \infty}$, so that the finite volume approximation of the initial datum by $f^{h}(0, \cdot)$ is actually of order 1 in $L^{1}$ with respect to $h$.

\section{NUMERICAL SIMULATIONS}

This section is devoted to the numerical study of two different phenomena: the convergence to equilibrium under the detailed balance condition and the gelation phenomenon, that is, the possible loss of matter during time evolution.

6.1. Detailed balance kernels and convergence to equilibrium. We assume that the coagulation and fragmentation coefficients fulfill the detailed balance condition: there exists a nonnegative function $M \in L_{1}^{1}\left(\mathbb{R}^{+} \times \mathbb{R}^{+}\right)$, such that

$$
a\left(x, x^{\prime}\right) M(x) M\left(x^{\prime}\right)=b\left(x, x^{\prime}\right) M\left(x+x^{\prime}\right), \quad\left(x, x^{\prime}\right) \in \mathbb{R}^{+} \times \mathbb{R}^{+} .
$$

Observe that this condition implies that $M$ is a stationary solution to (7), usually refered to as an equilibrium. An additional and interesting consequence of the detailed balance condition (63) is the existence of a Lyapunov functional $H$ [1] given by

$$
H(f):=\int_{\mathbb{R}^{+}} f(t, x)\left(\log \left(\frac{f(t, x)}{M(x)}\right)-1\right) .
$$

Indeed, any positive solution $f$ of the coagulation-fragmentation equation satisfies

$$
\begin{aligned}
\frac{d}{d t} H(f)= & -\frac{1}{2} \int_{\mathbb{R}^{+} \times \mathbb{R}^{+}}\left(a\left(x, x^{\prime}\right) f(t, x) f\left(t, x^{\prime}\right)-b\left(x, x^{\prime}\right) f\left(t, x+x^{\prime}\right)\right) \\
& \left(\log \left(a\left(x, x^{\prime}\right) f(t, x) f\left(t, x^{\prime}\right)\right)-\log \left(b\left(x, x^{\prime}\right) f\left(t, x+x^{\prime}\right)\right)\right) d x d x^{\prime} \\
\leq & 0 .
\end{aligned}
$$

Since any such solution $f$ decays the Lyapunov functional, convergence of $f$ towards the equilibrium state $M$ is expected. The first series of results proposed in this 
section is concerned with the observation of this trend to equilibrium. We choose kernels $a$ and $b$ as follows:

$$
a\left(x, x^{\prime}\right)=b\left(x, x^{\prime}\right)=\left(x x^{\prime}\right)^{1 / 2},
$$

so that

$$
M(x)=\exp (-x), \quad x \in \mathbb{R}^{+} .
$$

As an initial datum, we take

$$
f^{\text {in }}(x)= \begin{cases}2, & \text { if } 0 \leq x \leq 1 \\ 0, & \text { else }\end{cases}
$$

with $R=30, \Delta t=0.004$.
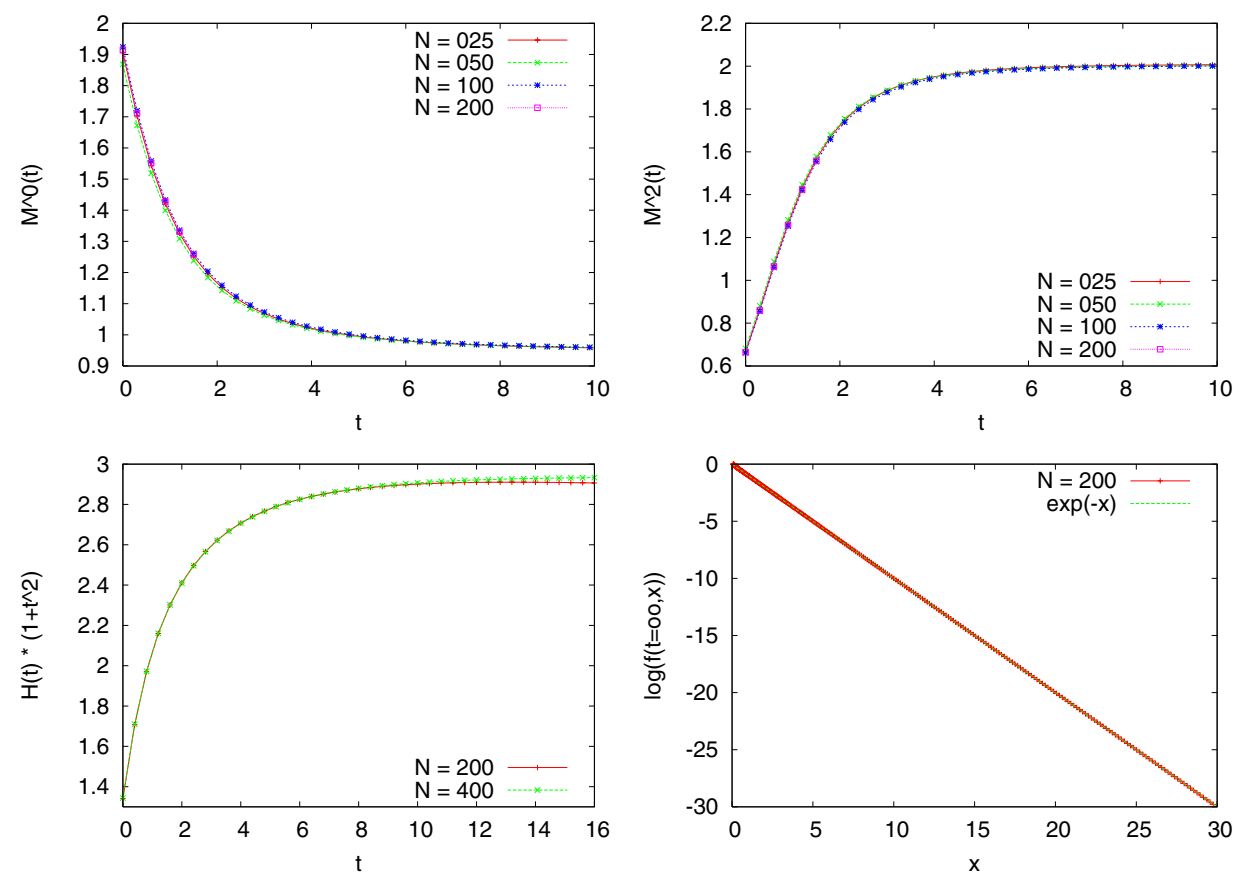

Figure 1. Evolution of the total number of particles $M_{0}$, the second moment of $f$, the functional $H(f(t))(1+t)^{2}$ and the stationary distribution $f(x)$ in $\log$ scale.

From a numerical point of view, some care is needed to compute the small $x$ behavior of the stationary state, taking into account that it also depends on its values for large $x$. Therefore, it is important to consider a suitable mesh in order to obtain an accurate numerical solution when $x$ is small but also for large $x$. We construct the following mesh:

$$
x_{i-1 / 2}= \begin{cases}e^{-6+\frac{7 i}{N}} & \text { for } 0 \leq i \leq N, \\ e+(R-e)\left(\frac{i-N}{N}\right)^{3 / 2} & \text { for } N+1 \leq i \leq 2 N,\end{cases}
$$

with $N=25,50,100$ and 200. This mesh satisfies the condition (13) and is extremely refined in the region close to the origin in order to describe with a lot of 
accuracy the solution in this region. On the other hand, the mesh $\Delta x_{i}$ is increasing for large $x$ in order to use a large enough truncature $R=30$ with few points.

In Figure 1, we report the evolution of the total number of particles $M_{0}$, the second moment of $f$, the Lyapunov functional $H(f)$ and the behavior of the asymptotic profile $f(+\infty, x)$. As expected, the total mass $M_{1}(t)$ remains constant throughout time evolution and the moments stabilize to a fixed value. In regard to the asymptotic profile, our numerical results are in fair agreement with the equilibrium $M(x)=\exp (-x)$, even when using few points $(N=25)$. Moreover, in view of the comparison between the exact steady state and the numerical solution for large time, we observe that using a non-uniform mesh allows to get a very good approximation in the region close to zero.

Concerning the rate of convergence, it seems that it is not an exponential rate: we plot the evolution of $H(f(t))(1+t)^{2}$, which may converge to a constant value. In a future work, we will investigate more accurately this behavior and expect to formulate some conjectures on the link between the rate of convergence and the kernels $a$ and $b$.

6.2. Occurrence of gelation. As already mentioned in the introduction and in 8, 19, 14, 20, 21, when the coagulation coefficient $a$ increases sufficiently rapidly with respect to the fragmentation kernel $b$ for large $x, x^{\prime}$, a runaway growth takes place and leads to the formation of a particle of "infinite mass" in finite time. As a consequence, the total mass $M_{1}$ decreases with time, and the gelation time $T_{\text {gel }}$ is defined by

$$
T_{g e l}:=\inf \left\{t \geq 0, \quad \int_{0}^{\infty} x f(t, x) d x<\int_{0}^{\infty} x f(0, x) d x\right\} \in[0,+\infty] .
$$

Then we say that gelation occurs if $T_{g e l}<+\infty$. Let us mention that this phenomenon is not yet completely understood mathematically. Indeed, it is expected that the gelation time $T_{g e l}$ also corresponds to the blow-up of high order moments (second order for instance), but there is no rigorous proof of that and it still constitutes a conjecture. An elementary proof that $T_{g e l}<+\infty$ was given in [21] when $a\left(x, x^{\prime}\right)=x x^{\prime}, b\left(x, x^{\prime}\right)=0$, and a central issue in the physical literature in the 1980 s was to figure out for which coefficients $a$ and $b$ the gelation time $T_{g e l}$ is finite. We restrict our discussion here to the model case

$$
a\left(x, x^{\prime}\right)=x^{\mu}\left(x^{\prime}\right)^{\nu}+x^{\nu}\left(x^{\prime}\right)^{\mu}, \quad b\left(x, x^{\prime}\right)=\left(x+x^{\prime}\right)^{\gamma}, \quad\left(x, x^{\prime}\right) \in\left(\mathbb{R}^{+}\right)^{2},
$$

with $0 \leq \mu \leq \nu \leq 1$ and $\gamma \in \mathbb{R}$. Putting $\lambda=\mu+\nu$, it follows from [9, Theorem 3.1] that there is a mass-conserving solution to (7) for any initial datum with a finite first order moment when $\gamma>\lambda-2$.

On the other hand, when $\gamma \in((\lambda-3) / 2, \lambda-2)$, it is proven in 9, Theorem 1.2] that if there exists a solution to (7), then gelation occurs when the initial first moment $M_{1}$ is large while there should be mass-conserving solutions when $M_{1}$ is small enough. Finally, gelation should occur for every non-zero solution when $\gamma<(\lambda-3) / 2$ [9, Section A.1].

We have first performed several computations to confirm the fact that the nonconservative truncation allows us to approximate as well mass-conserving solutions $(\gamma>\lambda-2)$ as gelation phenomenon $(\gamma<(\lambda-3) / 2)$. In these situations, the long time behaviour does not depend on the initial mass, but only on the relative proportion of coagulation and fragmentation phenomena. Not surprisingly, the solution is well approached by the scheme in this case and we prefer to give a detailed account 
of the more delicate situation where the long time behaviour strongly depends on the initial mass. Thus, the main purpose of the numerical simulations presented in this section is to observe numerically the intermediate regime $\gamma \in((\lambda-3) / 2, \lambda-2)$, which is, due to the dependence with respect to the initial datum, the most difficult to study.

We restrict ourselves here to the model case (65) with $\mu=\nu=\lambda / 2$; that is,

$$
a\left(x, x^{\prime}\right)=\left(x x^{\prime}\right)^{\lambda / 2}, \quad b\left(x, x^{\prime}\right)=\left(x+x^{\prime}\right)^{\gamma}, \quad\left(x, x^{\prime}\right) \in\left(\mathbb{R}^{+}\right)^{2},
$$

with $\lambda=5 / 2$ and $\gamma=0$. We take the following initial datum $f_{0}$ :

$$
f_{0}(x)=M_{1} \exp (-x), \quad x \in \mathbb{R}^{+} .
$$

Thus, the gelation phenomenon does take place when the initial mass $M_{1}$ is large enough and $T_{\text {gel }}<+\infty$ (see [9, Theorem 1.2]) and the authors conjecture that for a small initial mass $M_{1}$, there is a mass-conserving solution.

In Figure 2, we present our results for an initial mass $M_{1}=0.4$ and observe that gelation occurs at finite time $\left(T_{g e l} \in[0.925,1.05]\right)$. We see that the choice of the truncation (9), (10) and the scheme (16)-(21) provide a good estimate of the gelation phenomenon.

Next, the moments $M_{\ell}(t)$ are expected to blow up as $t \rightarrow T_{g e l}$ for $\ell \geq 2$. We compute numerical approximations of the solution to (7) with initial datum (66) for increasing values of the truncation parameter $R$. We define the moment of order $\ell \geq 0$ of the numerical approximation by

$$
\mathcal{M}_{\ell}^{h}\left(t^{n}\right)=\sum_{i=0}^{I^{h}} \Delta x_{i} x_{i}^{\ell} f_{i}^{n},
$$

and we plot the time evolution of the moments of order 0, 1 and 2 (see Figure 2). As expected, when $R$ is increasing, the number of particles $\mathcal{M}_{0}^{h}$ converge to a fixed value, whereas $\mathcal{M}_{2}^{h}$ is increasing to infinity when $R$ becomes larger and larger. In Figure 2, we represent the graph of some negative power of the second order moment, $\left[\mathcal{M}_{2}^{h}(t)\right]^{-1 / \alpha}$ with $\alpha=0.82$ and observe that when $R$ goes to infinity, it converges to a slightly concave curve (approaching a straight line) intersecting the axis at $t=1$, which means that $T_{g e l} \approx 1$ and

$$
\mathcal{M}_{2}(t) \approx \frac{C}{\left(T_{g e l}-t\right)^{\alpha}} .
$$

Thus, it seems to be clear that the gelation transition takes place in finite time and that there is a sudden growth of the moments of order 2 near the numerical gelation time. In particular, the growth rate increases for increasing values of $R$, which is seemingly good evidence for an occurrence of blow-up.

The fact that these moments decrease after the estimated gelation time is due to the finite length of the interval of computation $(0, R)$. Indeed, due to the nonconservative approximation, the amount of mass $\mathcal{F}_{I^{h}+1 / 2}^{n}$ is lost and high order moments then start to decrease with time.

In Figure 3, we also plot the evolution of the distribution function in log scale for different radius $R=1400$ and $R=6000$, we observe that on the interval $[0, R]$ both solutions are very close to each other. Moreover, around the gelation time $T_{\text {gel }}$, it seems that the tail of the distribution function changes its behavior and becomes algebraic. Unfortunately, it is very difficult to compute numerically how this tail behaves. 

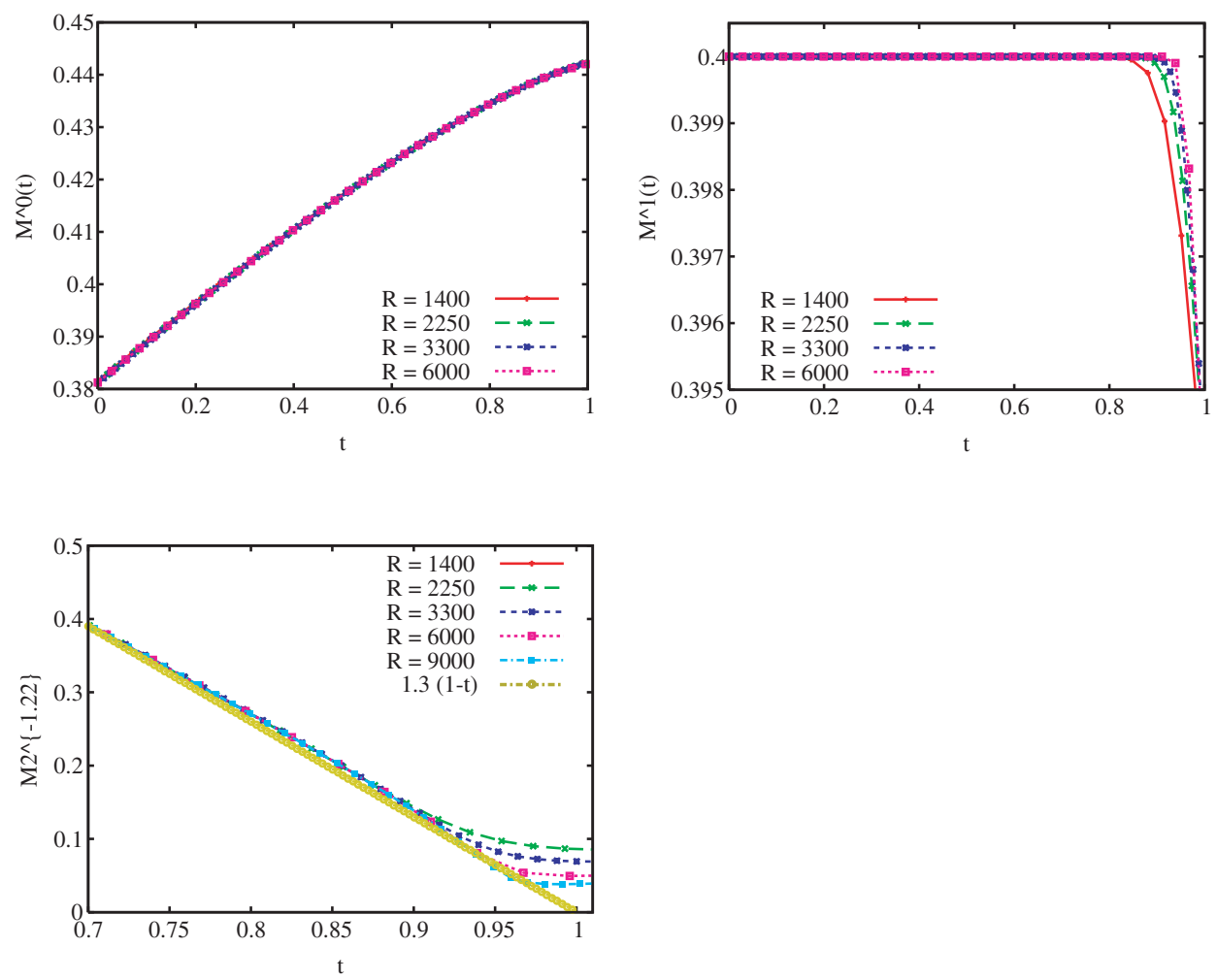

Figure 2. Initial mass $M_{1}=0.4$ : Evolution of the number of particles $M_{0}$, the total mass of particles $M_{1}$ and $\left[M_{2}(t)\right]^{-1 / \alpha}$ with $\alpha=0.82$.

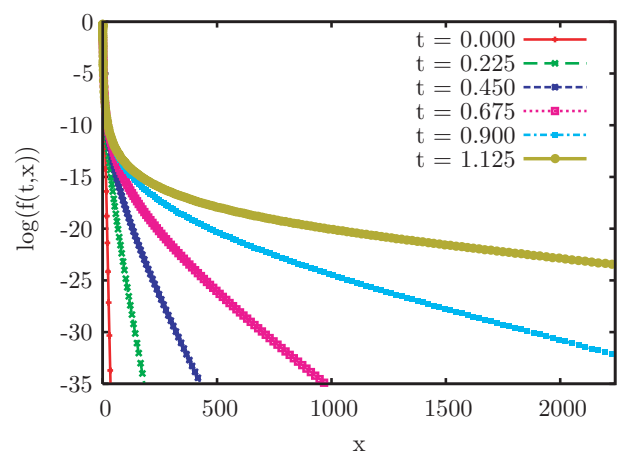

(a)

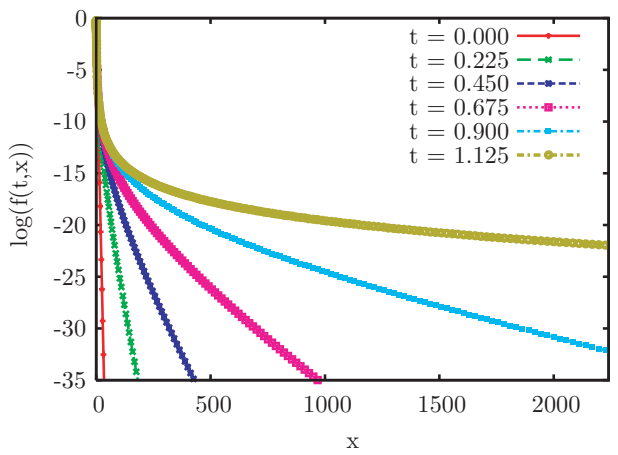

(b)

Figure 3. Initial mass $M_{1}=0.4$ : Evolution of the distribution $f(t, x)$ in $\log$ scale at time $t=0,0.225,0.45,0.675,0.9$ and 1.125 (after the estimated blow-up time) obtained with two different truncature (a) $R=1400$ and (b) $R=6000$. 

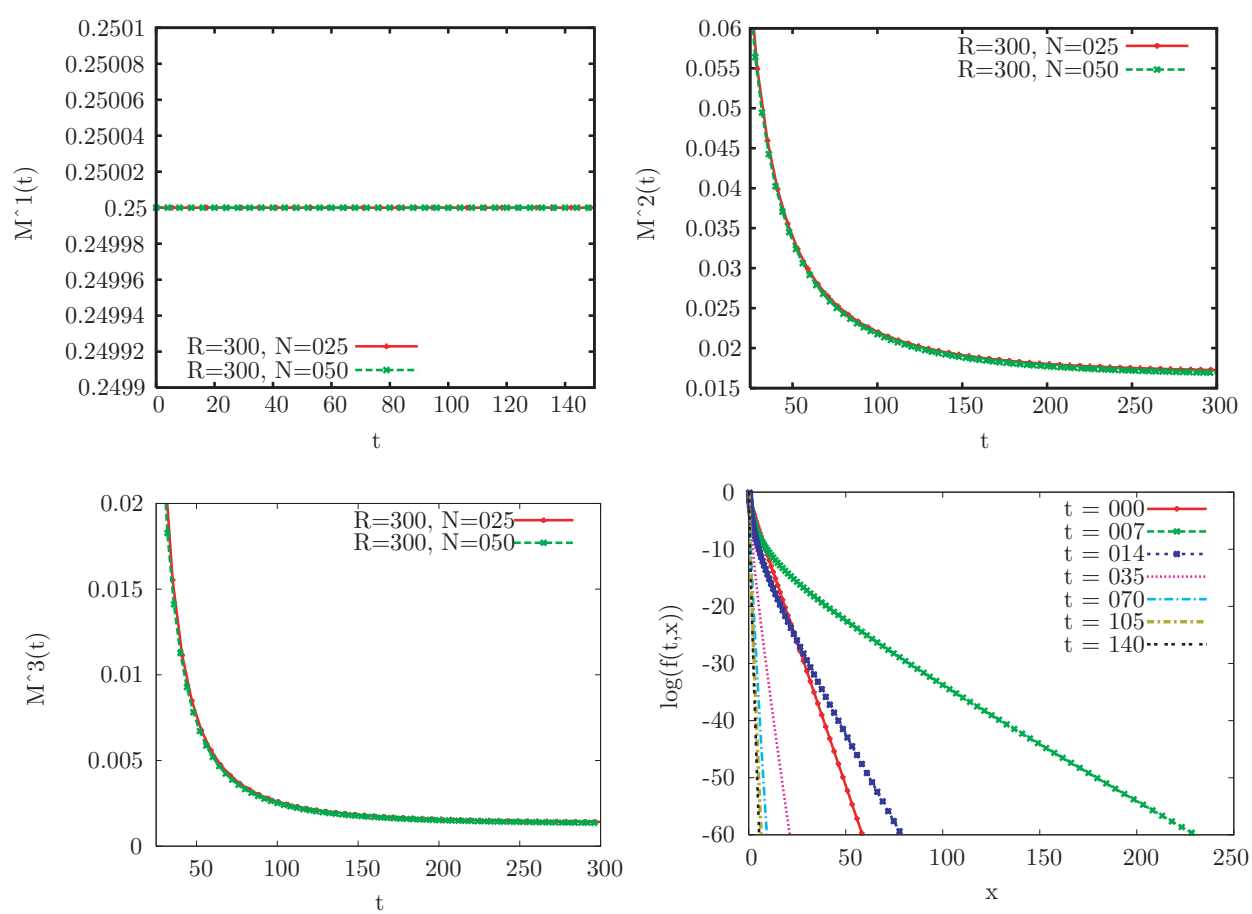

Figure 4. Initial mass $M_{1}=0.25$ : Evolution of the total mass of particles $M_{1}$, the second and third moments of $f$ for $t \geq 25$ and the distribution $f(t, x)$ in log scale at time $t=0,7,14,35,70,105$ and 140 .

On the other hand, we perform other computations (see Figure 4l) for a small initial mass $M_{1}=0.25$ and observe that in this case the solution is mass-conserving. Moreover, the numerical solution converges as time goes to infinity to an equilibrium (note that the detailed balance condition is not valid for these kernels $a$ and $b$ (65)): there are two different regimes in this case, the solution first spreads out and next concentrates itself to reach a steady state (see last pictures in Figure 4).

In a future work, it would be interesting to compute an approximation of the critical mass for which either the solution converges to a steady state or gelation occurs after a finite time. Up to now we focus on the qualitative behavior of the numerical solution.

\section{Conclusion}

This paper provides an extensive study of a discrete approximation of coagulation and fragmentation equations. The scheme first introduced for the discretization to the coagulation-fragmentation equation in [12] proves unexpectedly efficient in the description of gelation as well as long time behaviour of solutions to this model. In particular, it should be emphasized that mass conservation or dissipation is obtained in strong agreement with theoretical works.

The discretization is based on a divergence formulation, which (in association with the finite volume method) makes it well adapted to the observation of the 
time evolution of the total mass. Finally, numerical results seem to indicate the validity and the flexibility of the present approach that, in our opinion, will make deterministic schemes much more competitive with Monte Carlo methods in several situations for coagulation and fragmentation models.

\section{REFERENCES}

1. M. Aizenman And T.A. BAK, Convergence to equilibrium in a system of reacting polymers, Comm. Math. Phys. 65 (1979), pp. 203-230. MR.530150 (80d:80008)

2. H. Babovsky, On a Monte Carlo scheme for Smoluchowski's coagulation equation, Monte Carlo Methods Appl., 5 (1999), pp. 1-18. MR1684990 (99m:65010)

3. T. A. Bak And O. Heilmann, A finite version of Smoluchowski's coagulation equation, J. Phys. A, 24 (1991), pp. 4889-4893. MR1131259 (92g:82133)

4. F. P. DA Costa, A finite-dimensional dynamical model for gelation in coagulation processes, J. Nonlinear Sci., 8 (1998), pp. 619-653. MR 1650684 (2000a:82066)

5. R. L. Drake, A general mathematical survey of the coagulation equation, in Topics in Current Aerosol Research (Part 2), International Reviews in Aerosol Physics and Chemistry, Pergamon Press, Oxford, UK, (1972), pp. 203-376.

6. A. Eibeck And W. Wagner, An efficient stochastic algorithm for studying coagulation dynamics and gelation phenomena, SIAM J. Sci. Comput., 22 (2000), pp. 802-821. MR1784826 (2001e:82037)

7. L. D. Erasmus, D. Eyre, and R. C. Everson, Numerical treatment of the population balance equation using a Spline-Galerkin method, Computers Chem. Engrg., 8 (1994), pp. 775-783.

8. M. Escobedo, S. Mischler, And B. Perthame, Gelation in coagulation and fragmentation models, Comm. Math. Phys., 231 (2002), pp. 157-188. MR.1947695 (2003m:82053)

9. M. Escobedo, Ph. Laurençot, S. Mischler, and B. Perthame, Gelation and mass conservation in coagulation-fragmentation models, J. Differential Equations, 195 (2003), pp. 143-174. MR2019246 (2004k:82069)

10. R. Eymard, T. Gallouët And R. Herbin, Finite volume methods, in Handbook of numerical analysis, Vol. VII, 713-1020, Handb. Numer. Anal., VII, North-Holland, Amsterdam, 2000. MR 1804748 (2002e:65138)

11. F. Filbet AND Ph. LAUREnÇOT, Mass-conserving solutions and non-conservative approximation to the Smoluchowski coagulation equation, Arch. Math. (Basel) 83 (2004), pp. 558-567. MR2105334 (2005h:82081)

12. F. Filbet And Ph. LaUrençot, Numerical Simulation of the Smoluchowski Coagulation Equation, SIAM J. Sci. Comput. Vol. 25 (2004), pp. 2004-2028. MR2086828(2005i:65222)

13. F. Guins, A Monte Carlo approach to the Smoluchowski equations, Monte Carlo Methods Appl., 3 (1997), pp. 313-326. MR 1604836

14. I. JEON, Existence of gelling solutions for coagulation-fragmentation equations, Comm. Math. Phys., 194 (1998), pp. 541-567. MR.1631473 (99g:82056)

15. Ph. LaurenÇOt, On a class of continuous coagulation-fragmentation equations, J. Differential Equations, 167 (2000), pp. 145-174. MR1793195 (2001i:82044)

16. Ph. Laurençot and S. Mischler, From the discrete to the continuous coagulationfragmentation equations, Proc. Roy. Soc. Edinburgh Sect. A, 132 (2002), pp. 1219-1248. MR $1938720(2003 \mathrm{j}: 82056)$

17. C. LÉCOT AND W. WAGNER, A quasi-Monte Carlo scheme for Smoluchowski's coagulation equation, Math. Comp. 73 (2004), pp. 1953-1966. MR2059745 (2005c:82084)

18. M. H. LEE, On the validity of the coagulation equation and the nature of runaway growth, Icarus, 143 (2000), pp. 74-86.

19. R.J. LeVeque, Numerical methods for conservation laws, Second edition, Lectures in Mathematics ETH Zürich, Birkhäuser Verlag, Basel, 1992. MR1153252 (92m:65106)

20. F. Leyvraz, Existence and properties of post-gel solutions for the kinetic equations of coagulation, J. Phys. A, 16 (1983), pp. 2861-2873. MR715741 (85h:82030)

21. F. Leyvraz And H. R. Tschudi, Singularities in the kinetics of coagulation processes, J. Phys. A, 14 (1981), pp. 3389-3405. MR639565 (84h:80012)

22. J. Makino, T. Fukushige, Y. Funato, and E. Kokubo, On the mass distribution of planetesimals in the early runaway stage, New Astronomy, 3 (1998), pp. 411-417. 
23. G. Menon and R. L. Pego, Approach to self-similarity in Smoluchowski's coagulation equations, Comm. Pure Appl. Math. 57 (2004), pp. 1197-1232. MR2059679 (2005i:82051)

24. H. TANaka, S. InABA, And K. NAKAZA, Steady-state size distribution for the self-similar collision cascade, Icarus, 123 (1996), pp. 450-455.

25. R.M. ZiFf AND E.D. MCGRADY, The kinetics of cluster fragmentation and depolymerisation, J. Phys. A 18 (1985), pp. 3027-3037. MR814641(87a:82046)

Institut de Mathématiques de Toulouse, Université Toulouse III, 118, Route de NarBonne 31062 Toulouse CEDEX 09, France

E-mail address: bourgade@mip.ups-tlse.fr

Université Lyon, Université Lyon 1, CNRS, UMR 5208 - Institut Camille Jordan, 43 Boulevard du 11 Novembre 1918, F-69622 Villeurbanne cedex, France

E-mail address: filbet@math.univ-lyon1.fr 